\title{
Suppressing UPR-dependent overactivation of FGFR3 signaling ameliorates SLC26A2-deficient chondrodysplasias
}

\author{
Chao Zheng ${ }^{\mathrm{a}, 1}$, Xisheng Lin ${ }^{\mathrm{a}, 1}$, Xiaolong Xu ${ }^{\mathrm{a}, 1}$, Cheng Wang ${ }^{\mathrm{b}}$, Jinru Zhou ${ }^{\mathrm{a}}$, Bo Gao ${ }^{\mathrm{a}}$, Jing Fan ${ }^{\mathrm{a}}$, Weiguang Lu ${ }^{\mathrm{a}}$, \\ Yaqian $\mathrm{Hu}^{\mathrm{a}}$, Qiang Jie ${ }^{\mathrm{c}, 2}$, Zhuojing Luo ${ }^{\mathrm{a}, \mathrm{d}, 2}$, Liu Yang ${ }^{\mathrm{a}, \mathrm{d}, *, 2}$ \\ a Institute of Orthopedic Surgery, Xijing Hospital, Fourth Military Medical University, Xi'an, China \\ b School of Biomedical Sciences, University of Hong Kong, Hong Kong, China \\ c Department of Orthopedic Surgery, HongHui Hospital, Xi'an Jiaotong University, College of Medicine, Xi'an, China \\ d Medical Research Institute, Northwestern Polytechnical University, Xi'an, China
}

\section{A R T I C L E I N F O}

\section{Article history:}

Received 3 December 2018

Received in revised form 3 January 2019

Accepted 7 January 2019

Available online 23 January 2019

\section{Keywords:}

SLC26A2

Achondrogenesis type $1 \mathrm{~B}$

Atelosteogenesis type 2

Collagen secretion

FGFR3 signaling

\begin{abstract}
A B S T R A C T
Background: Mutations in the SLC26A2 gene cause a spectrum of currently incurable human chondrodysplasias. However, genotype-phenotype relationships of SLC26A2-deficient chondrodysplasias are still perplexing and thus stunt therapeutic development

Methods: To investigate the causative role of SLC26A2 deficiency in chondrodysplasias and confirm its skeletonspecific pathology, we generated and analyzed $s l c 26 a 2^{-1-}$ and Col2a1-Cre; slc26a $2^{f / f l}$ mice. The therapeutic effect of NVP-BGJ398, an FGFR inhibitor, was tested with both explant cultures and timed pregnant females.

Findings: Two lethal forms of human SLC26A2-related chondrodysplasias, achondrogenesis type IB (ACG1B) and atelosteogenesis type II (AO2), are phenocopied by slc26a2 $2^{-1-}$ mice. Unexpectedly, slc26a2 ${ }^{-1-}$ chondrocytes are defective for collagen secretion, exhibiting intracellular retention and compromised extracellular deposition of ColII and ColIX. As a consequence, the ATF6 arm of the unfolded protein response (UPR) is preferentially triggered to overactivate FGFR3 signaling by inducing excessive FGFR3 in slc26a2 $2^{-1-}$ chondrocytes. Consistently, suppressing FGFR3 signaling by blocking either FGFR3 or phosphorylation of the downstream effector favors the recovery of $s l c 26 a 2^{-/-}$cartilage cultures from impaired growth and unbalanced cell proliferation and apoptosis. Moreover, administration of an FGFR inhibitor to pregnant females shows therapeutic effects on pathological features in slc26a2 $2^{-1-}$ newborns. Finally, we confirm the skeleton-specific lethality and pathology of global SLC26A2 deletion through analyzing the Col2a1-Cre; slc26a $2^{f / f l}$ mouse line.

Interpretation: Our study unveils a previously unrecognized pathogenic mechanism underlying ACG1B and AO2, and supports suppression of FGFR3 signaling as a promising therapeutic approach for SLC26A2-related chondrodysplasias.

Fund: This work was supported by National Natural Science Foundation of China $(81871743,81730065$ and 81772377).

(C) 2019 The Authors. Published by Elsevier B.V. This is an open access article under the CC BY-NC-ND license (http://
\end{abstract} creativecommons.org/licenses/by-nc-nd/4.0/).

\section{Introduction}

Sulfation is a crucial post-translational modification for secretory biomolecules such as carbohydrates [1], steroid hormones [2] and tyrosine of proteins [3], while functional research on its effects is far from exhaustive. Notably, altered components of the sulfation pathway induce evident skeletal deformities in mouse models by disrupting

\footnotetext{
* Corresponding author at: Institute of Orthopaedics, Xijing Hospital, The Fourth Military Medical University, No. 127, Changle West Road, Xi'an, Shaanxi Province, China. E-mail address: yangliu@fmmu.edu.cn (L. Yang).

1 Chao Zheng, Xisheng Lin and Xiaolong Xu contributed equally.

2 Liu Yang, Zhuojing Luo and Qiang Jie are corresponding authors.
}

normal cellular uptake and metabolism of sulfates in chondrocytes [4-10]. Being the most upstream component in the sulfation pathway, SLC26A2 functions as the ubiquitously expressed sulfate transporter on the cell membrane and enables intracellular delivery of inorganic sulfate $[11,12]$. Thus far, over 55 mutations have been identified in the SLC26A2 gene and led to five inherited skeletal diseases with varying clinical severity, including achondrogenesis type IB (ACG1B), atelosteogenesis type II (AO2), diastrophic dysplasia (DTD), recessive multiple epiphyseal dysplasia (rMED) and dysplastic spondylolysis [13-15].

Previous studies carried out by Antonio Rossi and his coworkers with $d t d$ mice, a loss-of-function mutation knock-in mouse strain, explain the pathogenesis of DTD with a longstanding theory of 


\section{Research in context}

\section{Evidence before this study}

Over 55 mutations have been identified in the human sulfate transporter SLC26A2 gene and led to five inherited skeletal diseases with varying clinical severity, ranging from two lethal forms of achondrogenesis type IB (ACG1B) and atelosteogenesis type II (AO2) to other milder types including diastrophic dysplasia (DTD). Importantly, these SLC26A2-deficient chondrodysplasias are currently incurable due to still perplexing pathogenesis. Although a longstanding pathogenic theory of proteoglycan (PG) undersulfation was established from several studies with $d t d$ mice carrying a DTD-causing mutation in the SLC26A2 gene, it has been reported that the level of PG undersulfation did not absolutely correlate with the clinical severity of SLC26A2-related chondrodysplasias, suggesting other causative factors may jointly regulate phenotypic outcome in addition to undersulfated PGs. Particularly, sulfation is a crucial post-transcriptional modification for secretory proteins, and substrates of sulfation are far more than PGs in cartilage matrix. Indeed, abnormal extracellular deposition of collagens has been noticed in both patients with ACG1B and $\mathrm{AO} 2$ and $d t d$ mice, which warrants further investigation.

\section{Added value of this study}

We have studied ACG1B and AO2, two most severe forms of SLC26A2-related chondrodysplasias, by globally and conditionally deleting SLC26A2 in mice. A previously unrecognized causative role of SLC26A2 ablation in defective collagen secretion is highlighted in our study, which consequently triggers the ATF6 arm of the unfolded protein response (UPR) to over-activate FGFR3 signaling, one of the most profound inhibitory pathways regulating chondrocyte growth. Inspired by these findings, we further address a promising therapeutic approach targeting FGFR3 signaling to alleviate pathological hallmarks of ACG1B and AO2 in mice. This work on sulfate transporter SLC26A2 could be a springboard to understand the more complex role of sulfation in skeletal development and diseases than hitherto assumed.

\section{Implications of all the available evidence}

Our study provides a promising therapeutic target by revealing the causative role of overactivated FGFR3 signaling in impaired cartilage growth so that many clinically well-tested FGF receptor inhibitors could be repurposed to treat SLC26A2-deficient chondrodysplasias. Besides, our findings also indicate that targeting UPR-dependent signaling pathways could become an attractive approach to finely modulate UPR actions in other stressrelated diseases.

proteoglycan (PG) undersulfation [4,16-19]. However, this theory becomes less applicable when confronting the full repertoire of SLC26A2related chondrodysplasias. A growing body of evidence indicates that neither the residual sulfate uptake function of mutant SLC26A2 nor the level of PG undersulfation absolutely correlates with the clinical severity of SLC26A2-related skeletal conditions, ranging from the lethal ACG1B and AO2 to the milder DTD and rMED, suggesting that other unknown factors may also contribute to and vary the severity of phenotypes [20-24]. This perplexing genotype-phenotype relationship complicates therapeutic development and renders SLC26A2-related chondrodysplasias currently incurable.

Notably, the altered composition of collagen in the extracellular matrix (ECM) of cartilage growth plates has been observed in both ACG1B or AO2 patients and dtd mice [17,25,26]. Whether the decreased expression, defective secretion or accelerated degradation of collagen should be held responsible is, however, overlooked in that context. The collagens are a family of the ECM structural proteins that are highly enriched in connective tissues to fulfil their function as the major tensile element [27]. Among 27 types of collagens to date, ColII/IX/X/XI are almost exclusively expressed in the cartilage, and mutations of these collagens have been characterized in various human osteochondrodysplasias $[27,28]$. Ample evidence has indicated that many of the mutations in collagen genes cause inappropriate folding and accumulation of collagen proteins in the endoplasmic reticulum (ER) [29]. The retention of secretory proteins inside the ER induces a cellular condition known as the ER stress and triggers a set of adaptive mechanisms together referred to as the unfolded protein response (UPR), which underlies the pathogenesis of UPR-related chondrodysplasias [30,31]. Three classical arms of sensors control the UPR: activating transcription factor 6 (ATF6), inositol-requiring enzyme $1 \alpha$ (IRE1 $\alpha$ ) and protein kinase RNA-like ER kinase (PERK) [32]. While the activated UPR initially favors the recovery of proteostasis, it also paradoxically promotes apoptosis when cells are already severely damaged or fail to adapt to the chronic stress [33,34]. Particularly, UPR sensors have been reported to manipulate chondrocyte development and maturation by selectively transactivating chondrocyte-regulatory signaling molecules and transcription factors, such as parathyroid hormone 1 receptor (Pth1r), Indian hedgehog (Ihh), fibroblast growth factor 21 (FGF21), Sox9 and Runt-related transcription factor 2 (Runx2) [30,35-37], thereby functioning as discrete UPR signaling modules. Therefore, targeting UPR-dependent signaling pathways could become an attractive approach to finely modulate UPR actions in stressed chondrocytes.

In this study, we show that global deletion of SLC26A2 in mice causes perinatal lethality and a distinctive skeletal phenotype intermediate between ACG1B and AO2. Surprisingly, collagen secretion is defective in slc26a2 ${ }^{-/-}$chondrocytes, which consequently triggers the ATF6 arm of the UPR. In an ATF6-dependent manner, the fibroblast growth factor receptor 3 (FGFR3) signaling is substantially enhanced and dominates the pathogenesis of SLC26A2 deficiency among other chondrocyteregulatory pathways. Indeed, suppression of FGFR3 signaling evidently alleviates impaired cartilage growth of both explant cultures and slc26a2 $2^{-1-}$ newborns. Finally, we exclude the redundancy of SLC26A2 with other sulfate transporters in chondrocytes and confirm a direct causative role of SLC26A2 deficiency in skeletal deformities by analyzing Col2a1-Cre; slc26a $2^{f l / f l}$ mice. Collectively, our study demonstrates a previously unrecognized mechanism underlying ACG1B and AO2 and thus provides a novel therapeutic strategy.

\section{Materials and methods}

\subsection{Animals}

To generate $s l c 26 a 2^{f l f l}$ mice, mouse genomic fragments consisting of homology arms together with conditional knockout region were amplified from BAC clone and sequentially assembled into a targeting vector containing recombination sites and selection markers (Fig. S1C). The linearized vector was introduced into ES cells (C57BL/6) via electroporation. We gained 55 drug-resistant clones in total and finally confirmed six correctly targeted ES clones by Southern blotting (Fig. S1E), of which some clones were selected for blastocyst microinjection to generate chimeric mice. By crossing founders with Flp-deleter mice, we confirmed germ-line transmission of the floxed allele and obtained slc26a $2^{f l+}$ mice without the neo cassette. slc26a2 $2^{f l f l}$ mice were generated by

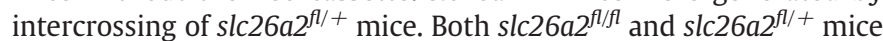
were viable and fertile and exhibited no apparent phenotypic changes or modification of behavior. The slc26a2-LoxP mouse line was genotyped by PCR using primers flanking LoxP site (5'-GCA ACA CTA TCT CTC TGC TTG GCC T- $3^{\prime}$ and 5'-ACC ACT AAG GAT TCT CCC GTG CAT$\left.3^{\prime}\right)$, and the results were analyzed as demonstrated in Fig. S1B. PCR 
screening for slc26a2 null allele was performed with primers flanking the coding region of exon 3 and 4 (5'-GCA ACA CTA TCT CTC TGC TTG GCC T-3' and 5'-AAC TTG CCC AGT TAC CAG GAA GAT-3'). Other used mice, including Vasa-Cre [38] and Col2al-Cre [39], were genotyped as previously reported respectively. Potential influences of mice genetic background were minimized by crossing mice lines involved in this study with wild-type C57BL/ 6 mice for $>9$ generations before any evaluation. Animal care and experiments were performed in accordance with protocols approved by the Ethics in Animal Research Committee of the Fourth Military Medical University.

\subsection{Skeletal preparation and Safranin O staining}

For whole-mount skeletal staining, neonates were collected immediately after birth and fixed in 95\% ethanol overnight at room temperature following evisceration. Samples were then placed in acetone overnight at room temperature and submerged in cartilage staining solution containing $0.03 \%(w / v)$ Alcian blue, $80 \%$ ethanol and $20 \%$ acetic acid overnight. Initial wash was performed by several changes of $70 \%$ ethanol to destain the cartilage. To better visualize the cartilage morphology, washing was ended before slc26a2 ${ }^{-1-}$ and Col2a1-Cre; slc26a $2^{f l / f l}$ cartilage was completely destained. Ossified tissues were stained by Alizarin red solution containing $0.005 \%(\mathrm{w} / \mathrm{v})$ Alizarin red in $1 \%(\mathrm{w} / \mathrm{v}) \mathrm{KOH}$ for $4 \mathrm{~h}$ at room temperature and sequentially placed at $4{ }^{\circ} \mathrm{C}$ overnight to slow down the staining. Samples were placed in $50 \%$ glycerol solution containing $1 \%(\mathrm{w} / \mathrm{v}) \mathrm{KOH}$ to remove excessive stains. Images of the stained skeleton were finally captured under a dissecting microscope utilizing bright field optics. For Safranin O staining, embryos were collected following euthanization of timed pregnant females and fixed overnight in 4\% paraformaldehyde. Tissues were embedded in OCT compound (Leica), cryosectioned at $8 \mu \mathrm{m}$ and stained with Safranin 0 and fast green as previously described [6].

\subsection{EdU incorporation and TUNEL assay}

Methods used in the study have been previously described with minor modifications [40]. In brief, cell proliferation was evaluated using EdU labelling assay. Timed pregnant females were intraperitoneally injected with $250 \mu \mathrm{g}$ of EdU (Invitrogen) per $10 \mathrm{~g}$ of body weight two or seven hours before euthanization. Hindlimbs of embryos were dissected, fixed overnight in $4 \%$ paraformaldehyde and embedded in OCT compound (Leica). EdU in tibial cryosections was detected using the Click-iT EdU Alexa Fluor 488 Imaging Kit (Invitrogen). Apoptotic cells were detected in situ on tibial cryosections by TUNEL assay using the In Situ Cell Death Detection Kit (Roche) according to the manufacturer's instructions.

\subsection{In-situ hybridization}

In-situ hybridization was performed on tibial paraffin sections with $\left[{ }^{35} \mathrm{~S}\right] \mathrm{UTP}-$ labeled riboprobes according to the previously described protocol [30]. Probes used in the study have been described previously: Sox9 and Col2a1 [30]; Col1a1, Col10a1 and MMP13 [40]; Runx2 [41]; Ihh, PTHrP receptor, Ptch1 and Gli1 [42].

\subsection{Immunostaining}

Immunofluorescence on tibial cryosections was essentially performed as previously described [30]. The primary antibodies used were anti-COL2A1 (Abcam, ab34712, 1:100), anti-COL10A1 (from Kathryn Song Eng Cheah, University of Hong Kong, 1:500), antiCOL9A1 (Bioworld Technology, BS6945, 1:50), anti-COL11A1 antibody (Abcam, ab64883, 1:100), anti-ATF6 antibody (Abcam, ab37149, 1:100), anti-ATF4 antibody (Abcam, ab31390, 1:100), anti-GRP78 BiP antibody (Abcam, ab21685, 1:100), anti-XBP1 antibody (Abcam, ab37152, 1:100), anti-phospho- Erk1/2 antibody (Cell Signaling
Technology, 4370, 1:200), anti-phospho-Stat1 antibody (Cell Signaling Technology, 9167, 1:200). Alexa Fluor 594 conjugate of Concanavalin A (Invitrogen) was used to colocalized with collagens. For ColII/IX/XI antigen retrieval, sections were treated with $2 \mathrm{mg} / \mathrm{ml}$ pepsin (Sigma Aldrich) for $10 \mathrm{~min}$ at $37{ }^{\circ} \mathrm{C}$. For ColX antigen retrieval, sections were digested with $2 \mathrm{mg} / \mathrm{ml}$ hyaluronidase (Sigma Aldrich) for $20 \mathrm{~min}$ at $37{ }^{\circ} \mathrm{C}$. The primary antibodies were detected with appropriate Alexa Fluor-conjugated secondary antibodies (Abcam). All sections were mounted with ProLong Gold Antifade Mountant with DAPI (Invitrogen) and visualized using a fluorescent microscope (Zeiss).

\subsection{Primary chondrocyte and cartilage explant culture}

For primary culture of chondrocytes, rib cartilage was isolated from E18.5 embryos and digested with $3 \mathrm{mg}$ per $\mathrm{ml}$ collagenase $\mathrm{D}$ (Roche) solution for $40 \mathrm{~min}$ at $37{ }^{\circ} \mathrm{C}$ under $5 \% \mathrm{CO}_{2}$ in a thermal incubator. Tissue fragments were agitated for several times to detach soft tissues and transferred into $0.5 \mathrm{mg}$ per ml collagenase D solution overnight at 37 ${ }^{\circ} \mathrm{C}$. Digestion solution was retrieved, filtered through an $8 \mu \mathrm{m}$ cell strainer and then centrifuged for $10 \mathrm{~min}$ at $400 \mathrm{~g}$. The pellet was resuspended with DMEM (Sigma Aldrich) supplemented with $10 \%$ fetal bovine serum (Gbico), $2 \mu \mathrm{M}$ L-GIn, $50 \mathrm{U}$ per ml penicillin and $0.05 \mathrm{mg}$ per $\mathrm{ml}$ streptomycin. Rib chondrocytes were seeded at the density of $25 \times 10^{3}$ cells per $\mathrm{cm}^{2}$ and cultured under sterile conditions at $37^{\circ} \mathrm{C}$ under $5 \% \mathrm{CO}_{2}$. For metatarsal culture, metatarsal explants were isolated from E17.5 embryos under a dissecting microscope and carefully placed into the pre-warmed 48-well plates containing $150 \mu \mathrm{l}$ of BGJb medium (Gibco) supplemented with $0.2 \% w / v$ BSA, $5 \mu$ g per $\mathrm{ml}$ L-ascorbic acid phosphate, $1 \mathrm{mM} \beta$-glycerophosphate, $0.05 \mathrm{mg}$ per $\mathrm{ml}$ gentamicin and $1.25 \mu \mathrm{g}$ Amphotericin B. Metatarsals were cultured at $37{ }^{\circ} \mathrm{C}$ under $5 \%$ $\mathrm{CO}_{2}$ in the incubator. To modulate FGFR3 signaling in cultures, left metatarsals were incubated in the medium containing each or combined each two of 500 nM NVP-BGJ398 (Selleck), 2 MM SCH772984 (Selleck) or 50 ng per ml FGF-2 (PeproTech), whereas the right counterparts were used as controls. Tibias were isolated from E17.5 embryos and cultured at the liquid-air interface according to the previously described protocol [43]. Left tibias were exposed to 500 nM NVP-BGJ398 (Selleck), and right ones were used as untreated controls. The images of metatarsal and tibia explants were captured at the beginning (day 0 ) and on day 5 . The length was measured using Image J software.

\subsection{Western blot and $q P C R$}

For immunoblot analysis, cells were lysed by RIPA buffer and centrifuged to extract total proteins. The protein concentration was measured using Pierce BCA Protein Assay Kit (Thermo Scientific). A certain amount of protein was mixed with loading buffer (Beyotime Biotechnology), boiled for $15 \mathrm{~min}$ and subjected to SDS-PAGE followed by transferring to PVDF membranes. Blots were probed with primary antibodies, including anti-ATF6 antibody (Abcam, ab37149, 1:1000), antiphospho-ERK1/2 antibody (Cell Signaling Technology, 4370, 1:1000), anti-ERK1/2 antibody (Cell Signaling Technology, 9102, 1:1000) and anti-FGFR3 antibody (Santa Cruz Biotechnology, sc-390,423, 1:1000). For qPCR, total RNA was extracted using MiniBEST Universal RNA Extraction Kit (TaKaRa). Reverse transcription was performed with PrimeScript RT Master Mix (TaKaRa). Synthesized cDNA was subjected to qPCR analysis using TB Green Premix Ex Taq II (TaKaRa). Primers used for qPCR to confirm Cre recombination were as follows: SLC26A2, 5'-AAG AGC AGC ATG ACC TCT CAC-3' (forward) and 5'-CTG CCT CAA GTC AGT GCC T-3' (reverse); GAPDH, 5'-AGG TCG GTG TGA ACG GAT TTG-3' (forward) and 5'-TGT AGA CCA TGT AGT TGA GGT CA-3' (reverse). 


\subsection{Analysis of FGFR3 signaling in primary chondrocytes}

When reaching $90 \%$ confluence, primary rib chondrocytes were treated with FGF-2 ( $50 \mathrm{ng}$ per $\mathrm{ml}$ ) for $5 \mathrm{~min}$ and lysed for Western blotting. For small interfering RNA (siRNA) transfection, primary chondrocytes reaching sub-confluence were incubated with $100 \mathrm{nM}$ siRNA mixed with siRNA-Mate Transfection Reagent (GenePharma) according to manufacturer's instructions. After 48-h transfection, chondrocytes were subjected to FGF-2 exposure and Western blot analysis as described above. The sequence of the siRNA targeting mouse ATF6 gene is 5'-GGA ACC AAA CCA GCA CCU UTT AAG GUG CUG GUU UGG UUC CTT- $3^{\prime}$. The control siRNA was a non-targeting sequence purchased from GenePharma Co. Ltd.

\subsection{Dual-luciferase reporter assay}

A promoter series were amplified from the mouse FGFR3 gene with the primers listed in Table S2 and cloned into pGL4.17 vector (Promega) to promote the expression of the luciferase. The gene fragment encoding ATF6 protein was amplified by PCR and cloned into pcDNA3. $1^{(+)}$vector (Invitrogen) using GTG GAA TTC atg gag tcg cct ttt agt ccg as the forward primer and TAG ACT CGA Gct act gca acg act cag gga tg as the reverse primer. MC3T3-E1 cells (ATCC, CRL-2594) were seeded at $1 \times 10^{5}$ cells per well in 24 -well plates. Transfection was performed using Lipofectamine 2000 (Invitrogen) according to the manufacturer's protocol when cells reached subconfluence. Each well contained $300 \mathrm{ng}$ tested constructs and $30 \mathrm{ng}$ pRL-SV40 (Promega) vector which served as an internal control. Twenty-four hours after transfection, luciferase assays were conducted using Dual-luciferase Reporter Assay System (Promega) following the manufacturer's instructions. The light units were measured with a luminometer (Promega Glomax 2020). Results were normalized to Renilla luciferase, presented as ratios of Luc/Renilla activity and acquired in triplicates from at least three different experiments.

\subsection{Drug treatment}

For in vivo NVP-BGJ398 treatment, pregnant females (E14.5) received daily intraperitoneal administration of NVP-BGJ398 (ApexBio Technology, $15 \mathrm{mg}$ per $\mathrm{kg}$ body weight, dissolved in $50 \mu \mathrm{l}$ DMSO) or vehicle ( $50 \mu \mathrm{l}$ DMSO) till the delivery. Under a surveillance camera, neonates were collected immediately after birth for further analyses.

\subsection{Statistical analysis}

Statistical analysis was performed with GraphPad Prism software, and the results were given as mean $\pm \mathrm{SD}$. Differences between experimental groups were assessed using the unpaired two-tailed Student's $t$ test or one-way ANOVA with Bonferroni post hoc test, and $p<.05$ was considered significant.

\section{Results}

3.1. slc26a2
phenotypes

To systemically ascertain the function of SLC26A2 in the cartilage and other tissues, we firstly constructed SLC26A2 total knockout mouse line using the Cre/loxP technology. Male slc26a $2^{f l / f l}$ mice, in which loxP sites flanked the whole coding region of exon 3 and 4 in the SLC26A2 gene as demonstrated in Methods, were in cross with female transgenic VasaCre mice, a germ cell-specific mouse transgenic Cre line permitting conversion of a floxed to a null allele in progeny [38], to generate Vasa-Cre; slc26a $2^{f l+}$ mice (Fig. S1A-B). Successful global Cre recombination was confirmed by PCR screening with primers flanking the coding region of exon 3 and 4 (Fig. 1A). An additional cross-breeding of Vasa-Cre; slc26a2 $2^{f l+}$ mice was conducted to generate SLC26A2 total knockout mice and to eliminate the transgene Cre. Only slc26a2 $2^{+/-}$and slc26a $2^{-/-}$mice were included for further studies. Analyses of quantitative real-time RT-PCR (qPCR) with previously reported SLC26A2expressing tissues indicated reduced mRNA levels of SLC26A2 in the samples from slc26a2 $2^{-1-}$ mice compared with those of $s l c 26 a 2^{f l f l}$ mice (Fig. 1B). The body size of slc26a2 $2^{+/-}$and slc26a2 $2^{-/-}$embryos diverged after E14.5 and became significantly different at E15.5 (Fig. 1C-D). Notably, the mesenchymal condensation is around E11.5-E12.5 in mice, which happens to precede the occurrence of morphological differences between slc26a2 $2^{+/-}$and slc26a2 $2^{-/-}$embryos [44,45]. All P0 slc26a2 $2^{-/-}$ newborns ( $>60)$ died immediately after birth without showing any respiratory movement of the thorax and manifested an overall look featured by short neck with thickened soft tissue, small chest, extremely short limbs and protuberant abdomen (Fig. 1C). Alcian blue/Alizarin red skeletal stains indicated multiple skeletal defects, including a global shrinkage in the size of both appendicular and axial, but to a less extent, skeleton; impaired ossification of tympanic and long bones; hypoplastic clavicles; small thoracic cage with shortened and somewhat broadened ribs; platyspondyly, accelerated and irregular ossification of vertebral bodies and premature closure of neurocentral synchondroses; typically bowed tibias (Fig. 1E-H and Fig. S2A-B). More importantly, these defects mostly characterize skeletal deformities of human ACG1B and $\mathrm{AO2}$, two neonatally lethal chondrodysplasias in the spectrum of SLC26A2-related skeletal disorders [46,47]. To further explain the neonatal death of slc26a2 $2^{-1-}$ mice, we performed $\mu \mathrm{CT}$ scanning and found no air in the thoracic cage of P0 slc26a2 $2^{-/-}$newborns, and histological analysis of lung sections showed signs of atelectasis (Fig. S2C-D). Skeletal preparation and rib cartilage sections showed that the rib cartilage was thicker but less densely populated by chondrocytes in P0 slc26a2 ${ }^{-/}$ - newborns than slc26a2 $2^{+/-}$littermate controls (Fig. S2E-F). Despite the ubiquitous expression of SLC26A2 in various tissues, no apparent overall phenotype was found in the kidney, heart and gastrointestinal tract of P0 slc26a2 ${ }^{-/-}$newborns (Fig. S2G). Taken together, hypoplasia of the thorax may contribute to the neonatal lethality of slc26a2 $2^{-/-}$neonates by causing respiratory disorders.

3.2. SLC26A2 deletion disrupts cartilage growth plate morphogenesis by inhibiting chondrocyte proliferation and hastening cell death

At the cellular level, in slc26a2 $2^{-1-}$ growth plates, a relatively minor decrease in the length of proliferating zone and the volume expanding of hypertrophic chondrocytes were observed at E14.5, and clearance of hypertrophic chondrocyte was slightly delayed at E15.5 compared with slc26a2 $2^{+/-}$littermate controls (Fig. S3A). We then focused on E18.5 when growth plate defects were most apparent in slc26a2 $2^{-/-}$ mice. Safranin O, a cationic dye showing affinity to polyanionic sulfated molecules, was used to reveal the sulfation degree of the ECM. Consistent with previous studies on human ACG1B and AO2 [48], the cartilage matrix was less intensely stained by Safranin $O$ in slc26a2 $2^{-1-}$ growth plates than slc26a2 $2^{+/-}$controls, which indicated matrix undersulfation (Fig. 1I). The morphology of slc26a2 ${ }^{-1-}$ growth plates was severely disrupted, showing shortened length, disorganized chondrocyte alignment and decreased cellularity (Fig. 1I-K). Chondrocyte columns were almost absent, and volume expanding of hypertrophic chondrocytes was inhibited in slc26a2 $2^{-1-}$ growth plates (Fig. 1I). We observed multiple cystic acellular areas in slc26a2 $2^{-/-}$growth plates among areas of relatively normal cellularity (Fig. 1I and Fig. S3B), which was consistent with the observation in cartilage sections from patients with ACG1B and $\mathrm{AO2}$ [47]. Interestingly, some of these areas were rimmed with chondrocytes harboring flattened nuclei (Fig. S3B). To investigate whether defective morphogenesis of slc $26 a 2^{-/-}$growth plates resulted from the altered balance between chondrocyte proliferation and apoptosis, we performed in vivo EdU labelling and TUNEL assay. Compared 
A

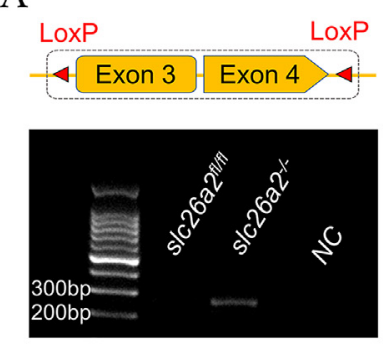

C

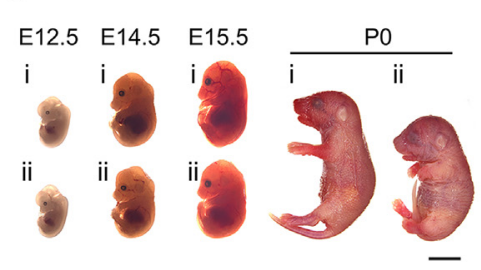

I
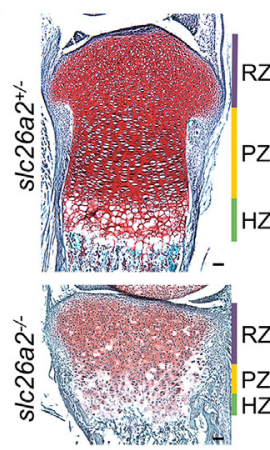

\section{J}

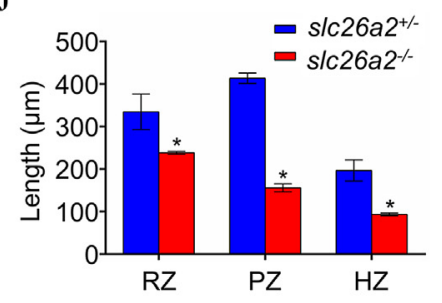

B

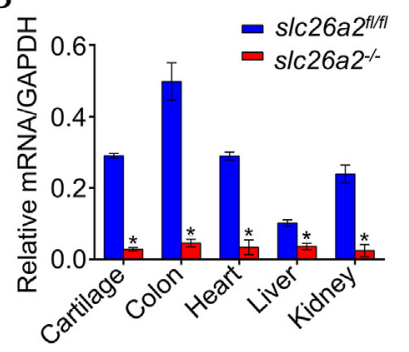

D

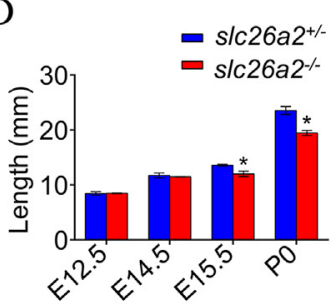

$\mathrm{E}$
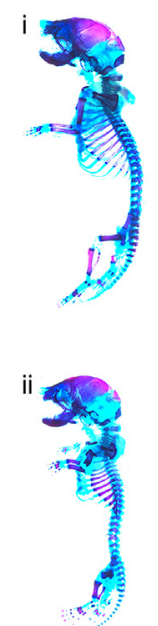

F
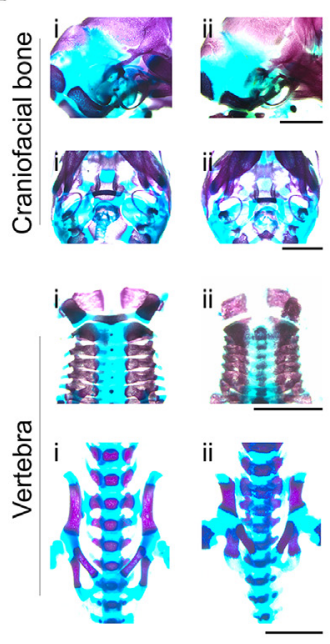

L

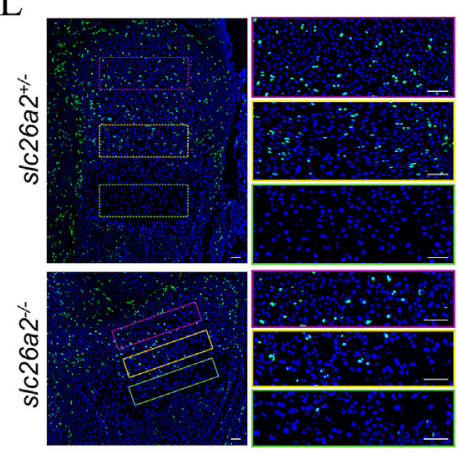

M

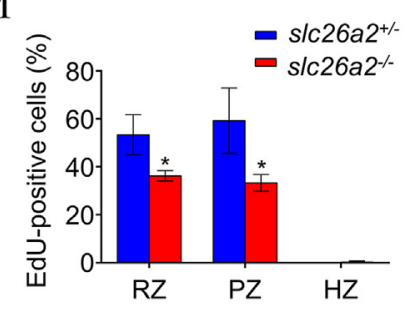

G

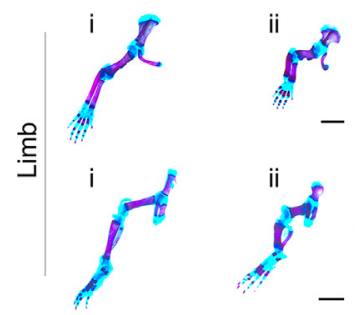

$\mathrm{H}$
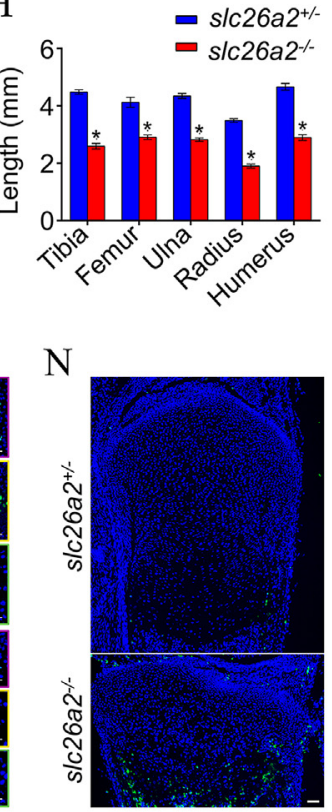

$\mathrm{O}$

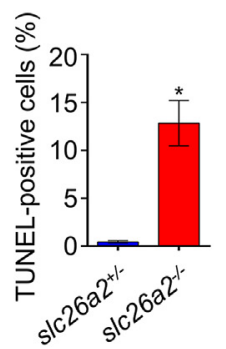

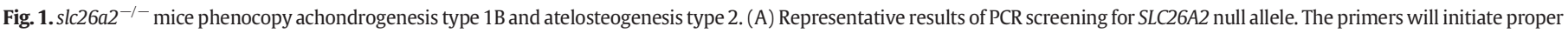

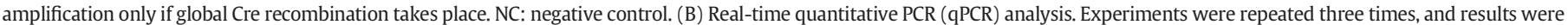

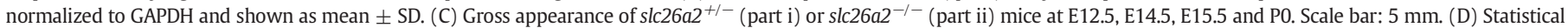

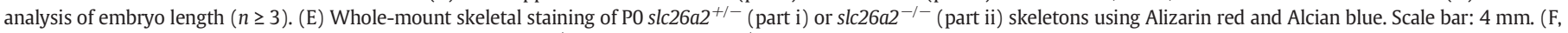

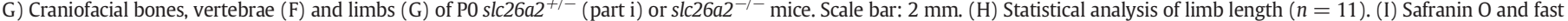

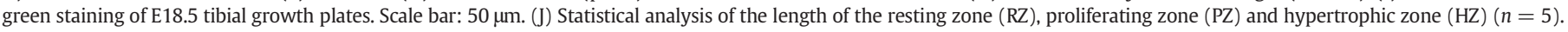

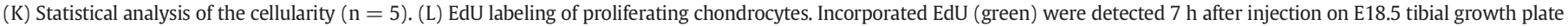

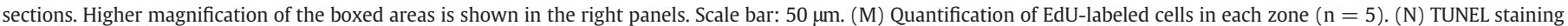

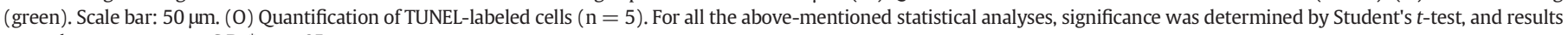
were shown as mean \pm S.D. ${ }^{*}: p<.05$.

with slc26a2 $2^{+/-}$littermate controls, the growth plates of E18.5 slc26a2 ${ }^{-1-}$ embryos exhibited a significant decrease in the number of EdU-labeled chondrocytes in the resting and proliferating zone in two and seven hours after EdU injection (Fig. 1L-M and Fig. S3C). Results from TUNEL staining on E18.5 tibial sections demonstrated that positive signals were only restricted to a few late-stage hypertrophic chondrocytes in slc26a2 ${ }^{+/-}$growth plates, whereas TUNEL-positive cells appeared in both proliferating and hypertrophic zones in slc26a2 $2^{-1-}$ growth plates (Fig. 1N). Notably, the number of TUNELpositive chondrocytes increased by more than ten times in slc26a2 $2^{-1-}$ growth plates than that of slc26a2 $2^{+/-}$littermate controls (Fig. 10).

\subsection{SLC26A2 regulates chondrocyte maturation and differentiation}

To determine whether disturbed morphogenesis of slc26a2 $2^{-1-}$ growth plates might reflect abnormalities of chondrocyte differentiation and maturation, we performed RNA in situ hybridization to locate and analyze different chondrocyte subpopulations. In slc26a2 ${ }^{+/-}$tibial growth plates at E15.5 and E18.5, early hypertrophic chondrocytes that abundantly expressed Col10a1 showed dramatically reduced expression of both Sox9, an early chondrocyte-specific marker, and Col2a1, a marker for proliferating and prehypertrophic chondrocytes (Fig. 2A-B). In slc26a2 $2^{-/-}$tibial growth plates, Col10a1-expressing 
A
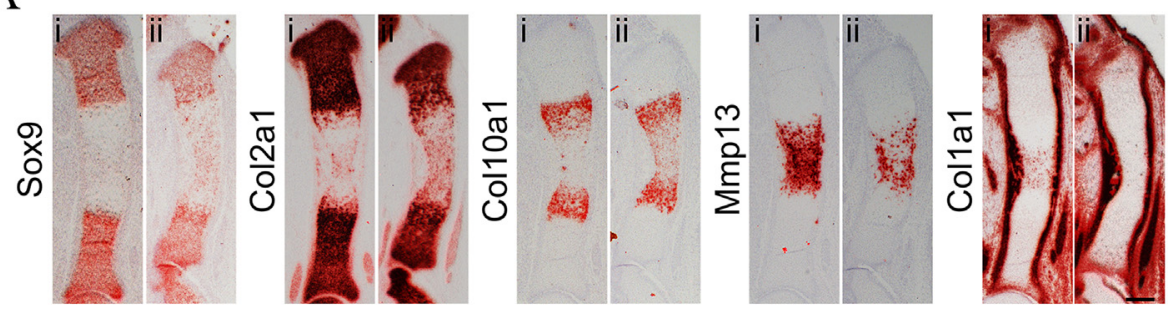

B

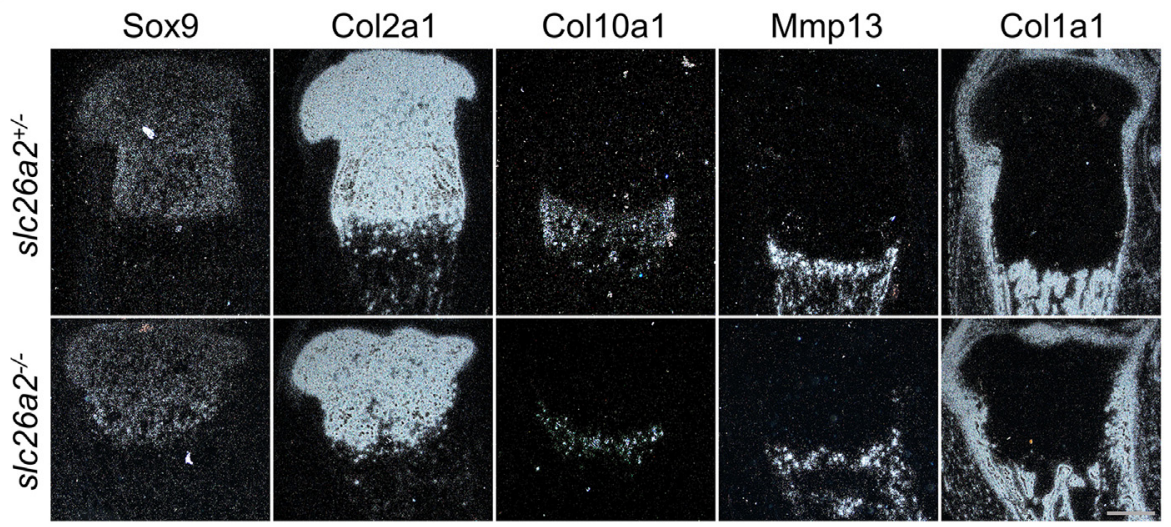

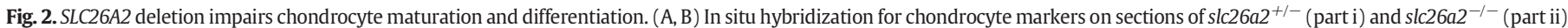
tibial growth plates at E15.5 (A) and E18.5 (B). Scale bar: $200 \mu \mathrm{m}$.

chondrocytes, however, still maintained a relatively higher level of Sox9 and Col2a1 expression, demonstrating delayed maturation of slc26a2 ${ }^{-/}$ - chondrocyte (Fig. 2A-B). Moreover, Col10a1-expressing range in E18.5 slc26a2 $2^{+/-}$and slc26a2 $2^{-1-}$ tibial growth plates evidently dwindled and were no longer comparable to that observed at E15.5 (Fig. 2A-B). Around E15.5, with the terminal differentiation of hypertrophic chondrocytes, vascularization and replacement of the cartilage with endochondral bones take place in the limb growth plate of mice [49]. As shown in Fig. 2A, MMP13, a marker for late hypertrophic chondrocytes and newly recruited osteoblasts, was highly expressed in the lower hypertrophic zone of E15.5 slc26a2 ${ }^{+/-}$tibial growth plates, exhibiting a complementary expression pattern with Col10a1. Conversely, in E15.5 slc26a2 ${ }^{-1-}$ counterparts, the expression of MMP13 was reduced, indicating that fewer hypertrophic chondrocytes reached terminal differentiation due to SLC26A2 deletion (Fig. 2A). We observed a certain number of late-stage hypertrophic chondrocytes expressing Col1a1, a marker also highly expressed by osteoblastic cells, in E15.5 slc26a $2^{+/-}$growth plates, whereas Col1a1 expression was almost missing in E15.5 slc26a2 ${ }^{-1-}$ growth plates (Fig. 2A). Furthermore, in E18.5 slc26a2 $2^{-1-}$ growth plates, reduced expression of MMP13 and Col1a1 was not only observed in the lower hypertrophic zone but also at the osteochondral junction where trabecular bones were formed (Fig. 2B). Consistently, we observed reduced trabecular bone formation and irregular vascular invasion at E18.5 slc26a2 $2^{-1-}$ osteochondral junctions compared with those of $s l c 26 a 2^{+/-}$littermate controls (Fig. S3D-E).

\section{4. slc26a2 $2^{-1-}$ chondrocytes are defective for collagen secretion}

PG undersulfation has been well documented in the context of SLC26A2 malfunction [4], whereas collagens, another major category of matrix elements actively secreted by chondrocytes, haven't been fully explored. We first examined ColII, the most abundantly expressed collagen in the growth plate [50], by performing immunostaining. In E18.5 slc26a2 ${ }^{+/-}$tibial growth plates, ColII was uniformly distributed in the extracellular space (Fig. 3A) and barely colocalized with fluorescent Concanavalin A (Fig. 3B), a common probe for endoplasmic reticulum localization [51]. Strikingly, the expression pattern of ColII was considerably altered in E18.5 slc26a2 ${ }^{-1-}$ tibial growth plates. Firstly, extracellular ColII was almost missing in places of acellularity, and only a few dim fluorescent signals could be detected around chondrocytes (Fig. 3A-B). Secondly, the dramatically increased ColII appeared to be intracellular and showed a massive colocalization with Concanavalin A, suggesting that ColII was retained in the ER due to SLC26A2 deletion (Fig. 3B). Thirdly, ColII retention seemed more drastic in the distal tibial growth plate than in the proximal (Fig. S4). Similarly, considerable immunofluorescent signals of ColIX were detected inside of slc26a2 $2^{-1-}$ growth plate chondrocytes rather than in the ECM and colocalized with Concanavalin A, which was less seen in slc26a2 ${ }^{+/-}$ chondrocytes (Fig. 3A-B). The immunostaining pattern of ColXI and ColX was comparable between slc26a2 $2^{-1-}$ and slc26a2 ${ }^{+/-}$growth plates in the aspect that both signals reached out into the ECM (Fig. 3A). However, the expression range of ColX was dwindled in slc26a2 $2^{-1-}$ growth plates compared with that of slc26a2 $2^{+-}$controls. Particularly, ColII, ColIX and ColXI were undetectable in those abovementioned "cystic acellular areas" (Fig. 3A). Consistent with the abnormal distribution of collagens revealed by immunofluorescence, transmission electron microscope (TEM) analysis of slc26a2 $2^{-1-}$ chondrocytes showed aberrant distension of the ER and reduction of fibril-like structures surrounding the chondrocytes, compared with slc26a2 $2^{+-}$controls (Fig. 3C and Fig. S5A). Massive intracellular accumulation of matrix-containing vesicles was observed in slc26a2 ${ }^{-/-}$ chondrocytes (Fig. S5B). Additionally, scanning electron microscope (SEM) analysis indicated disorganized and reduced scaly structures of cartilage matrix in slc26a2 ${ }^{-1-}$ growth plates (Fig. S5C).

\subsection{Activated UPR and enhanced FGFR3 signaling in slc26a2 $2^{-1-}$ chondrocytes}

Considering intracellularly aggregated collagens and the distended ER in slc26a2 $2^{-1-}$ chondrocytes, we further questioned whether the UPR was induced and performed immunofluorescence to detect UPR sensors in situ (Fig. 4A). Consistently, we observed an overexpression, 
A
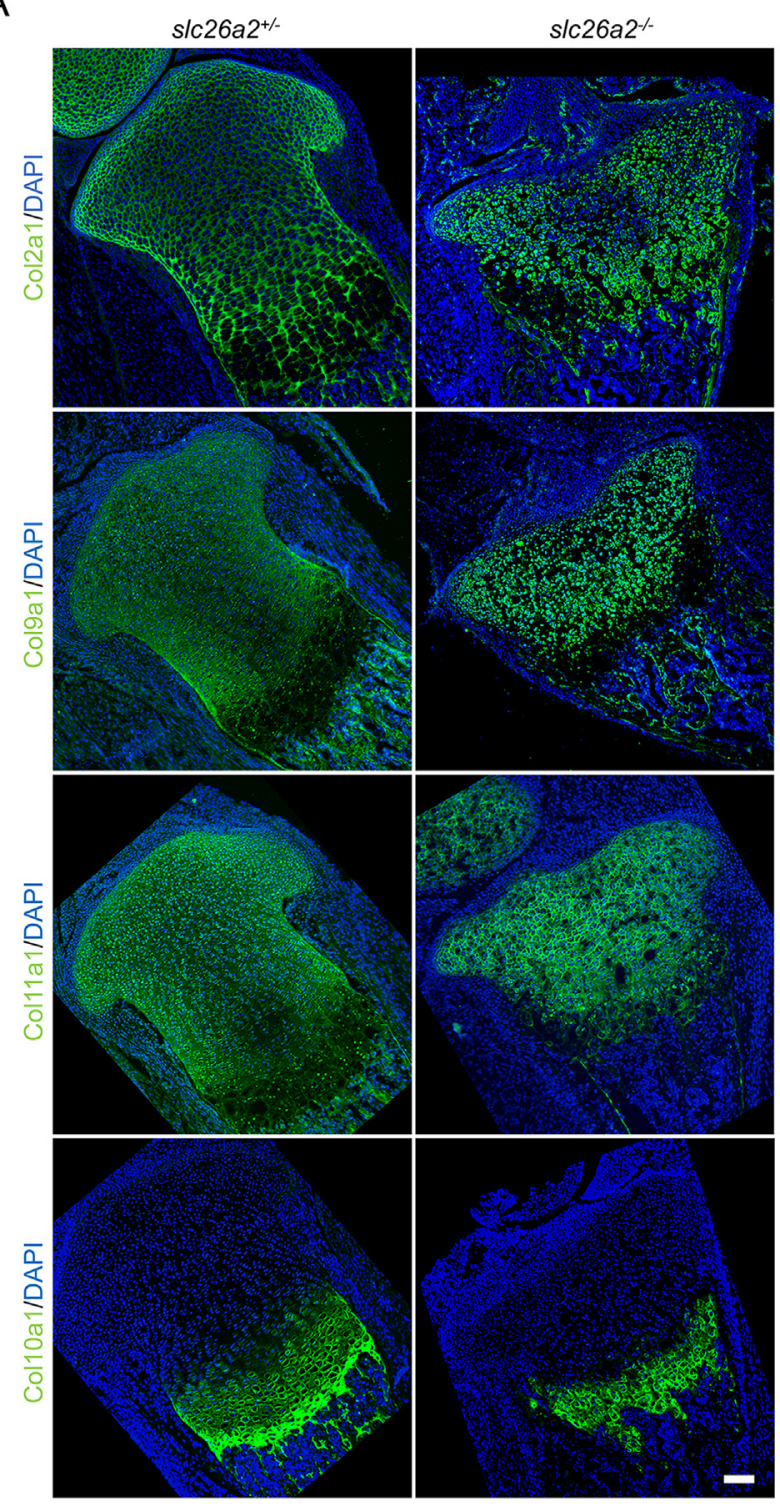

B

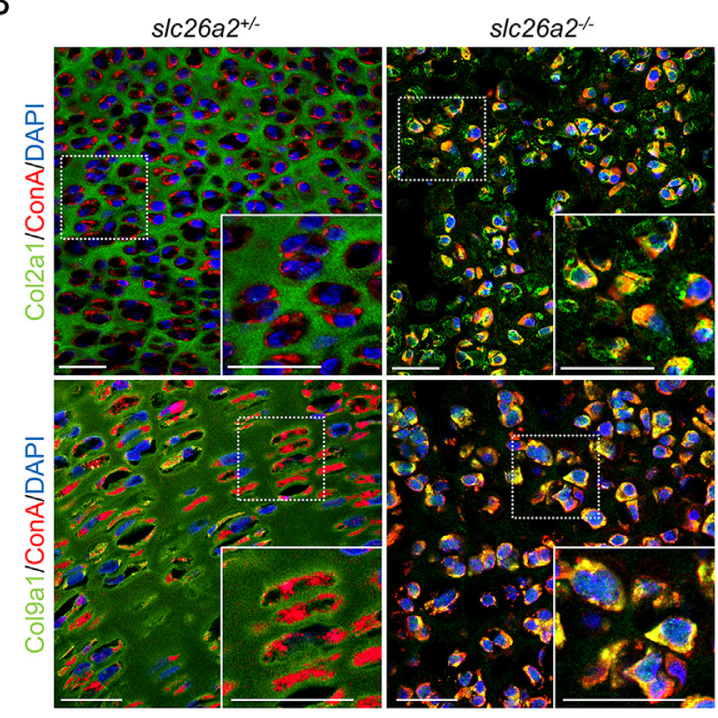

C

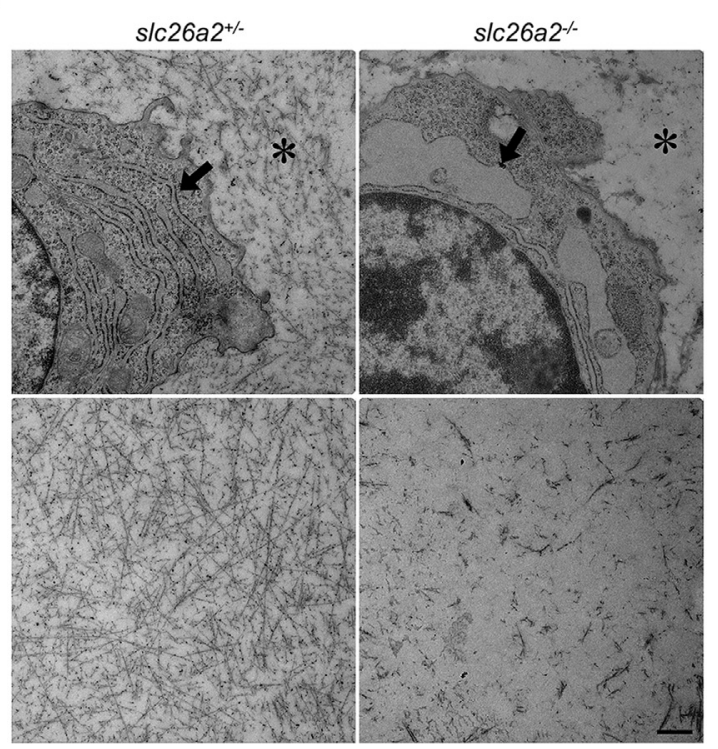

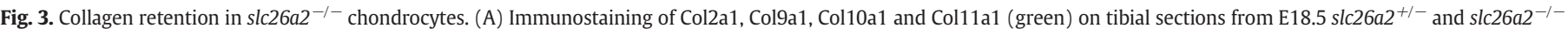

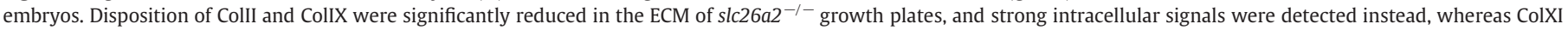

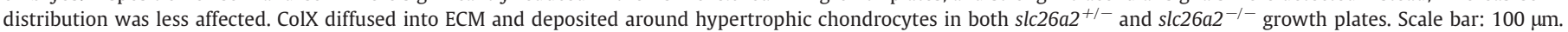

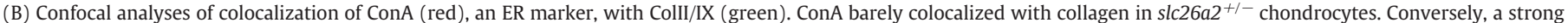

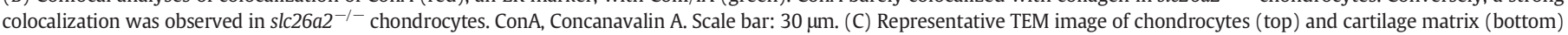

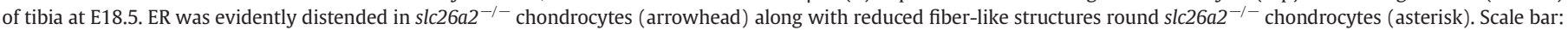
$500 \mathrm{~nm}$.

to various degree, of UPR readouts, including ATF4, BIP, CHOP, ATF6 and $\mathrm{XBP} 1$, in slc26a2 $2^{-/-}$proliferating chondrocytes compared with the slc26a2 $2^{+/-}$counterpart (Fig. 4A). Notably, among these UPR transducers, we found a preferentially upregulated expression of ATF6 along with a strikingly increased nucleus colocalization in slc26a2 $2^{-1-}$ proliferating chondrocytes, which was scarcely detected in slc26a2 $2^{+-}$proliferating chondrocytes (Fig. 4A). To ascertain potential downstream mediators of the ATF6 arm of the UPR, we examined signaling readouts, Ptch1, Gli1, Hip1, Ppr, Wnt5a, Wnt5b, Cyclin D1 and p-Smad2, of important growth factors regulating cartilage growth, Wnts, Ihh and TGFß, by carrying out in situ hybridization and immunostaining on sections of tibial growth plates (Fig. S6A). Compared with slc26a2 $2^{+/-}$littermate controls, we found patterns of these above-mentioned readouts roughly similar in slc26a2 $2^{-1-}$ growth plates (Fig. S6A). However, ERK1/2 and STAT1 as the putative effectors of FGFR3 signaling, one of the most profound inhibitory pathways during cartilage development, stood out for considerably enhanced phosphorylation in slc26a2 $2^{-1-}$ chondrocytes (Fig. 4B). Additionally, phosphorylated STAT1 (p-STAT1) was mainly detected in slc26a2 $2^{+1-}$ proliferating and pre-hypertrophic chondrocytes while signals of phosphorylated ERK1/2 (p-ERK1/2) were restricted to the hypertrophic zone (Fig. S6B). In slc26a2 $1-$ growth plates, p-ERK1/2 and p-STAT1 exhibited not only increased intensity but also an extended distribution throughout nearly all subpopulations of chondrocytes (Fig. 4C and Fig. S6B).

\subsection{Overactivated FGFR3 signaling in slc26a2 ${ }^{-1-}$ chondrocytes is ATF6- dependent}

To further investigate the molecular mechanism underlying SLC26A2 ablation, we cultured primary chondrocytes of rib cartilage from E18.5 slc26a2 ${ }^{+/-}$and slc26a2 $2^{-1-}$ embryos. Consistent with upregulated ATF6 and enhanced FGFR3 signaling indicated by immunostaining, 
A

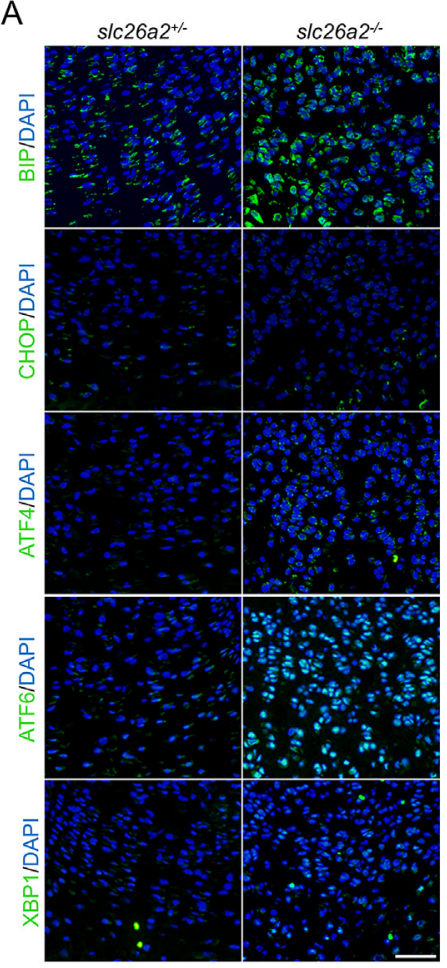

B

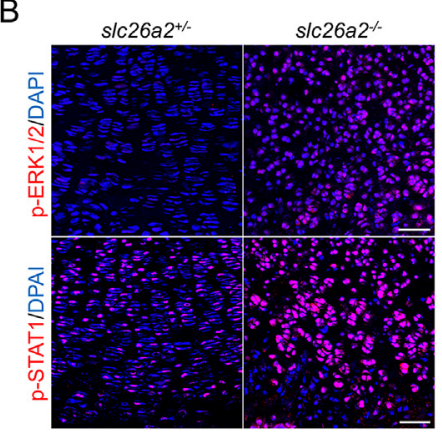

C

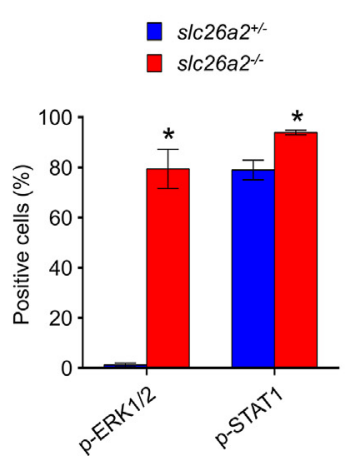

D

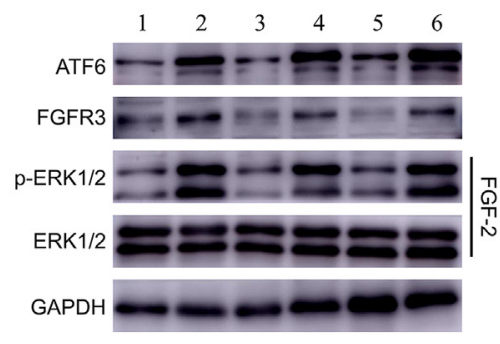

$\mathrm{E}$

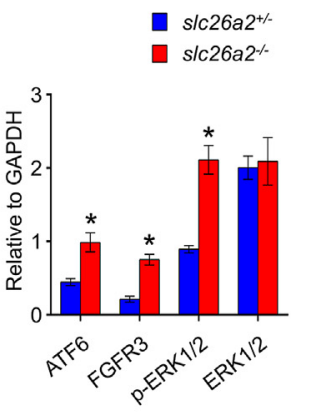

$\mathrm{F}$

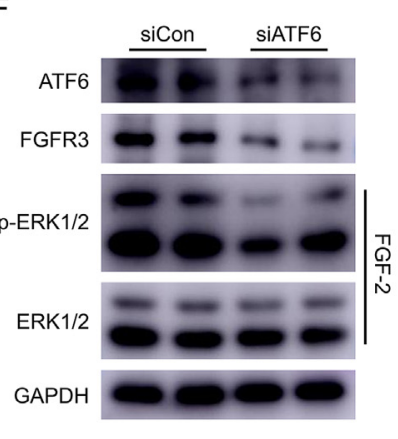

G

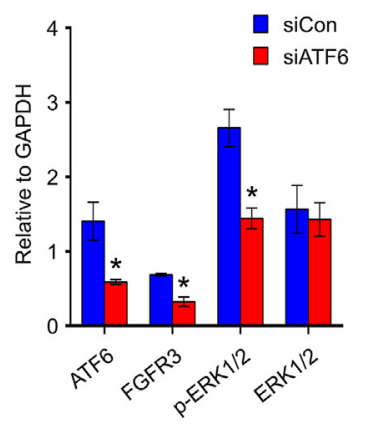

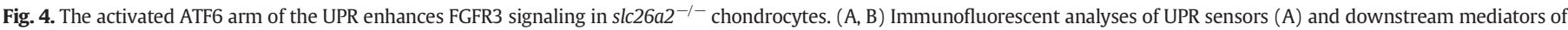

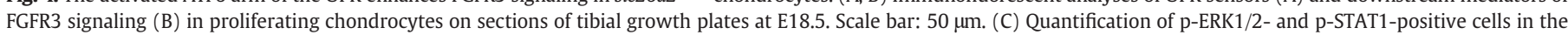

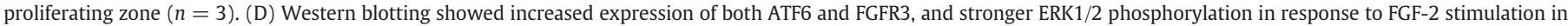

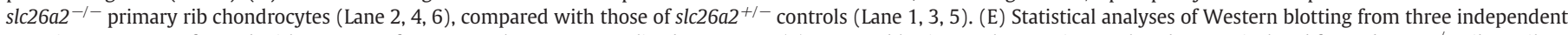

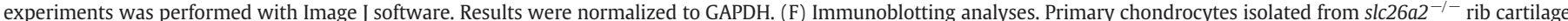

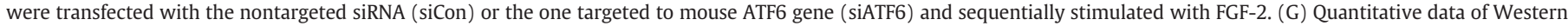
blotting from three independent experiments.

western blot analyses confirmed that both ATF6 and FGFR3 were increased at the protein level in primary slc26a2 ${ }^{-1-}$ chondrocytes, compared with those of slc26a2 $2^{+1-}$ controls (Fig. 4D-E). Given that the upregulation of FGFR3 proteins might sufficiently enhance the signaling, we, therefore, examined the FGF sensitivity of slc26a2 ${ }^{-1-}$ primary chondrocytes. To accomplish this, we stimulated chondrocytes with FGF-2 and used ERK1/2 phosphorylation as a readout for signaling activation [52]. As expected, slc26a2 ${ }^{-1-}$ chondrocytes showed a hypersensitivity to FGF2 by exhibiting a substantially stronger elevation of ERK1/2 phosphorylation than what was seen in slc26a2 $2^{+/-}$ chondrocytes (Fig. 4D-E). We next investigated whether increased expression of FGFR3 resulted from upregulation of ATF6 in slc26a2 $2^{-/-}$ chondrocytes by detecting FGFR3 expression after ATF6 knockdown (Fig. 4F-G). Immunoblots showed that endogenous ATF6 was downregulated when slc26a2 $2^{-/-}$chondrocytes were transfected with the siRNA targeted to mouse ATF6 gene (siATF6), compared to slc26a2 ${ }^{-1-}$ chondrocytes transfected with a nontargeted control siRNA (siCon). Importantly, FGFR3 expression together with the cellular response to FGF2 stimulation was significantly reduced along with ATF6 knockdown, compared with siCon transfection (Fig. 4F-G).

\subsection{ATF6 regulates transcription of FGFR3 through the responsive element in the first intron}

Given that ATF6 is a potent stress-related transcriptional factor $[53,54]$, we were prompted to test whether ATF6 can directly promote FGFR3 expression. We searched for ATF6 binding sites in the mouse FGFR3 gene employing precomputed ATF6 models in the TRANSFAC Public database and found 18 candidate sequences (Table S1). To include 8 predicted ATF6 binding sites, we split a total of 2887 bp regulatory region before the start codon ATG of mouse FGFR3 gene in three, namely -2270 to $-1320,-1320$ to $-102,-102$ to +616 (transcription start site $=+1$ ), and cloned each into a pGL4.17 vector for the promoter assay based on the dual-luciferase system (Fig. 5A-B). The relative luciferase activity of the $-102 /+616$ construct was significantly elevated when ATF6 was overexpressed, whereas no augmented luciferase activity was detected along with co-transfection of ATF6 and the other two tested constructs (Fig. 5B). Interestingly, the $-102 /$ +616 construct only contains one predicted binding sequence in intron I (TGAGTTGG, +137 from the transcription start site), suggesting direct transactivation of FGFR3 by ATF6 (Fig. 5A and Table S1). To further locate and confirm the regulatory potential of ATF6-response element, we selectively deleted $5^{\prime}$ cis-regulatory sequences between -110 and +616 to create a new panel of promoter constructs, including basal $c 1(-110 /+616), c 2(+145 /+616)$ and $c 3(-110 /+136,+145 /$ +616 ) (Fig. 5C). Absence of the predicted ATF6 binding site in $c 2$ and $c 3$ reduced the strong upregulation of luciferase activity observed with the co-transfection of $c 1$ and ATF6, although a fairly weak upregulation of luciferase activity was still detected when either $c 2$ or $c 3$ was cotransfected with ATF6, compared with mock controls (Fig. 5D). Moreover, mutation of the binding site, TGAGTTGG to TTTGTTGG, was sufficient to abolish transcriptional regulation by ATF6, as evidenced by completely failed ATF6 transactivation of luciferase reporters controlled by promoter $c 4(-110 /+616$ with above-mentioned mutations) (Fig. 5D).

\subsection{Targeting FGFR3 signaling modulates cartilage growth in vitro}

To confirm whether FGFR3 signaling contributes to impaired cartilage growth, we examined the growth of cultured metatarsal explants from E17.5 slc26a2 ${ }^{-1-}$ embryos with exposure to different regulatory reagents of FGFR3 signaling pathway. When stimulated with NVP- 

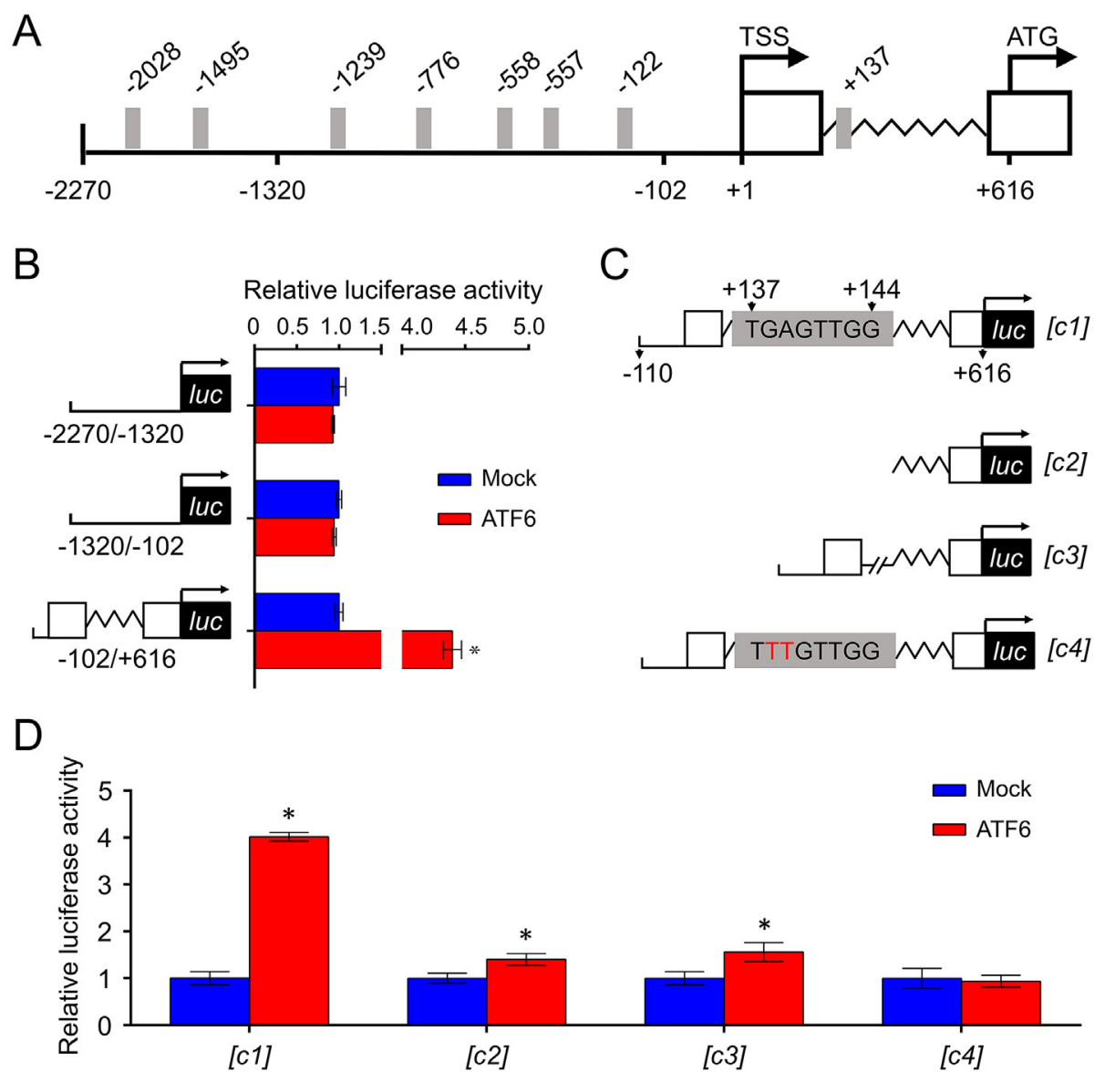

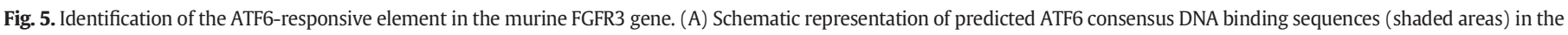

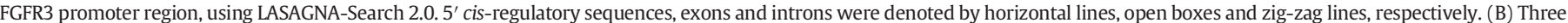

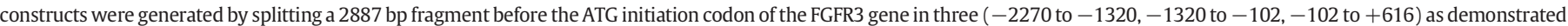

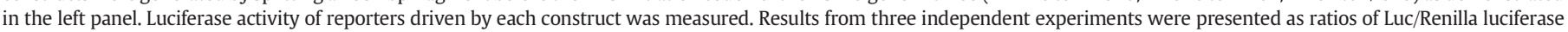

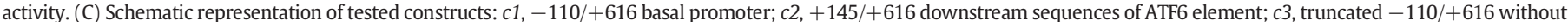

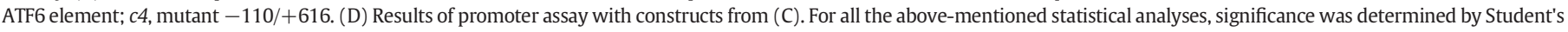
$t$-test, and results were shown as mean \pm SD. ${ }^{*}: p<.05$.

BGJ398, a selective inhibitor for FGFR3 [55], explant cultures exhibited significantly ameliorated defective elongation compared with the vehicle-treated (Fig. 6A-B). We also observed similarly improved growth of explants by inhibiting ERK1/2 kinase, a putative downstream effector of FGFR3 [56], with SCH772984 in comparison with control cultures (Fig. 6A-B). Next, we speculated that if hyperactive FGFR3 signaling was responsible for impaired cartilage growth caused by SLC26A2 deficiency, further activation of FGFR3 would worsen the phenotype. As expected, explants treated with FGF2 demonstrated further impaired growth than those treated with vehicle (Fig. 6A-B). Moreover, either SCH772984 or NVP-BGJ398 treatment was sufficient to alleviate the worsened growing retardation caused by FGF2 challenging (Fig. 6A-B). To further evaluate whether NVP-BGJ398 could restore uncoordinated cell proliferation and death in slc26a2 ${ }^{-1-}$ growth plates, we cultured E17.5 embryonic tibias from slc26a2 $2^{-1-}$ embryos. Consistent with our findings in cultured metatarsal explants, NVP-BGJ398 improved defective elongation compared with vehicle-treated cultures (Fig. 6C-D). Notably, we observed that NVP-BGJ398 treatment increased chondrocyte proliferation and decreased cell death in comparison with the vehicletreated, indicated by Ki67 immunostaining and TUNEL staining respectively (Fig. 6E-F).

\subsection{NVP-BGJ398 ameliorates skeletal defects of slc26a2 $2^{-1-}$ newborns}

Based on these encouraging in vitro data, we were prompted to test whether NVP-BGJ398 could ameliorate chondrodysplasia induced by
SLC26A2 ablation in vivo. To accomplish this, we treated pregnant females from $14.5 \mathrm{dpc}$ with intraperitoneal injections of NVP-BGJ398 (15 mg per $\mathrm{kg}$ body weight per day till delivery) and collected slc26a2 $2^{-1-}$ newborns for further analyses. Surprisingly, some extreme cases $(n=2)$ of all slc26a2 ${ }^{-/-}$newborns $(n=9)$ from pregnant females treated with NVP-BGJ398 could survive P0, still exhibiting aberrant respiratory movement, but fail to live through P1, whereas all slc26a2 ${ }^{-1-}$ newborns $(\mathrm{n}=9)$ from vehicle-treated pregnant females died immediately after birth (Fig. 7A). No noticeable phenotypic changes or modification of behavior were found in P0 slc26a2 ${ }^{+/-}$littermate newborns with NVP-BGJ398 treatment (data not shown). NVPBGJ398 partially rescued the limb shortening, including the humerus, radius, ulna, femur and tibia, in slc26a2 $2^{-1-}$ newborns from pregnant females treated with NVP-BGJ398 compared with slc26a2 $2^{-1-}$ newborns from vehicle-treated pregnant females (Fig. 7B, D). Histological analyses of the proximal tibial growth plates revealed that NVP-BGJ398 treatment significantly expanded the proliferating zone and increased the number of both discoid chondrocytes and well-aligned chondrocyte columns which were scarcely seen in growth plates of vehicle-treated controls (Fig. 7C, E). Besides, defective volume expansion of hypertrophy chondrocytes was improved in growth plates of slc26a2 $2^{-1-}$ newborns in NVP-BGJ398-treated group (Fig. 7C). In line with these favorable morphological changes, chondrocyte proliferation was significantly increased in NVP-BGJ398-treated slc26a2 ${ }^{-1-}$ growth plates, indicated by the increased number of Ki67-positive chondrocytes (Fig. 7F, H). Furthermore, NVP-BGJ398 treatment also prevented 
A

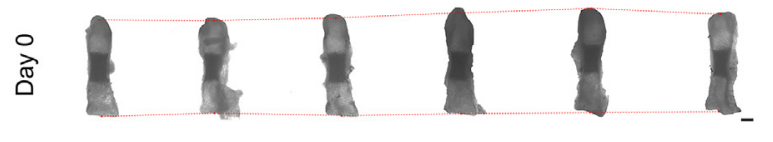

111118
B

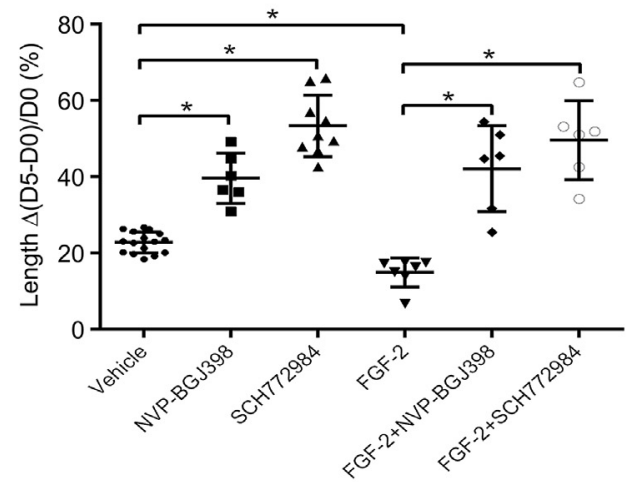

C

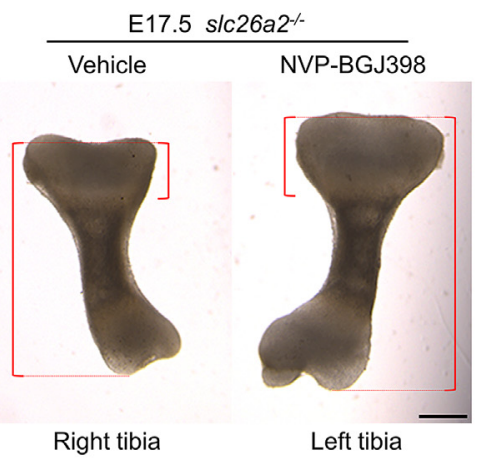

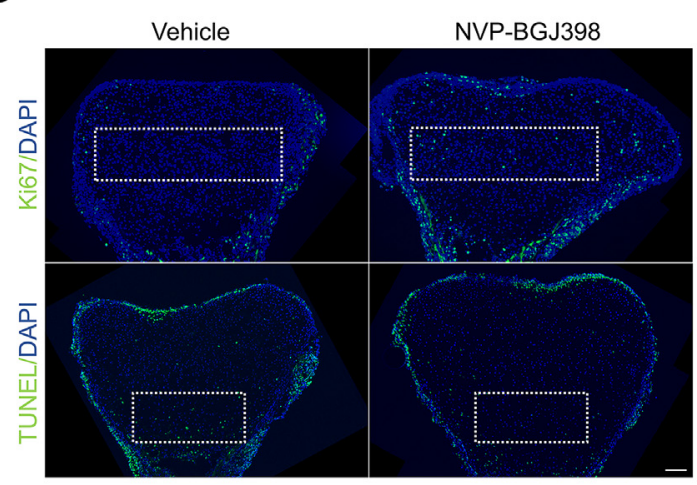

E

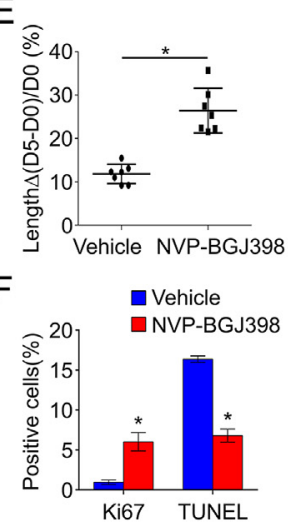

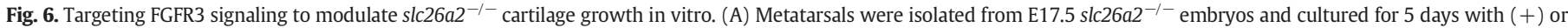

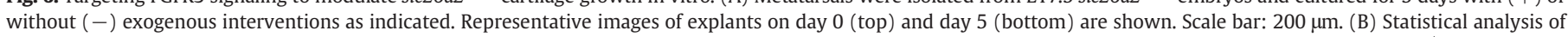

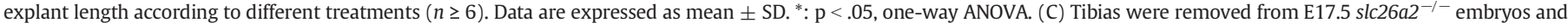

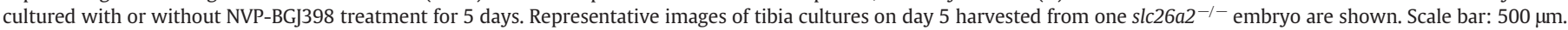

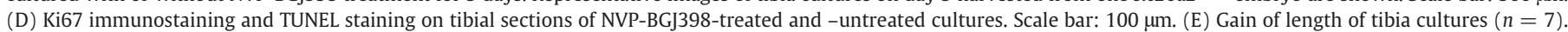

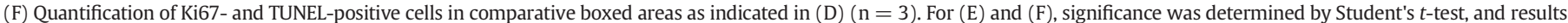
were shown as mean \pm SD. ${ }^{*}: \mathrm{p}<.05$.

apoptosis of slc26a2 $-1-$ chondrocytes, revealed by TUNEL staining on tibial sections of slc26a2 ${ }^{-/-}$neonates (Fig. 7F, H). To confirm whether NVP-BGJ398 efficiently tunes down FGFR3 signaling, we performed immunostaining of p-ERK $1 / 2$ on sections of tibial growth plates and observed a drastic lowering of ERK1/2 phosphorylation in growth plates of NVP-BGJ398-treated slc26a2 ${ }^{-/-}$newborns than those of vehicletreated slc26a2 ${ }^{-1-}$ controls (Fig. 7G, H). Collectively, these data suggest that NVP-BGJ398 treatment is effective in vivo to improve the long bone growth and coordinate cell death and proliferation by suppressing FGFR3 signaling in slc26a2 $2^{-/-}$chondrocytes.

\subsection{Col2a1-Cre; slc26a $2^{f l f l}$ mice recapitulate the phenotype of slc26a2 ${ }^{-/-}$ mice}

Lines of evidence have revealed potential roles of SLC26A2 in the pathogenesis of primary aldosteronism and development of ulcerative colitis and Crohn's disease and colon cancer, and even dtd mice showed abnormal aldosterone secretion, thereby justifying its ubiquitous nonskeletal expression [57-61]. Importantly, SLC26A2 is also expressed in cytotrophoblasts and considered to facilitate maternal-to-fetal delivery of sulfate $[62,63]$. It is possible that organ failures or deficient placental sulfate transport could lead to perinatal lethality and contribute to the skeletal phenotype. To confirm the direct role of SLC26A2 in cartilage, we generated conditional slc26a2 knockout mice by crossing Col2a1Cre transgenic mice with slc26a $2^{f l / f l}$ mice (Fig. S7A). Analysis of qPCR indicated that SLC26A2 was deleted in the cartilage without affecting its expression in other SLC26A2-expressing tissues of Col2a1-Cre; slc26a $2^{f l / f l}$ mice (Fig. 8A). Similar to slc26a2 $2^{-/-}$embryos, the body size of E12.5 Col2a1-Cre; slc26a2 $2^{f l / f l}$ embryos did not diverge from that of Col2a1-Cre; slc26a $2^{f l+}$ littermate controls whereas the difference became readily apparent at E15.5 (Fig. 8B and Fig. S7B). All Col2a1-Cre; slc26a $2^{f l f l}$ mice $(n>30)$ died immediately after birth and exhibited very similar skeletal disorders with what was seen in slc26a2 $2^{-/-}$mice, including shortening of long bones, hypoplasia of the thorax, premature fusion of vertebral ossification centers and defective ossification of tympanic bones (Fig. 8C-F). Histological analyses of E18.5 tibial growth plates indicated similar loss of Safranin O stains and morphological alterations, including decreased cellularity, poorly aligned columnar chondrocytes and insufficient volume expansion of hypertrophic chondrocytes (Fig. 8G). Likewise, analyses of Ki67 immunostaining and TUNEL staining showed significantly reduced chondrocyte proliferation and increased apoptosis in E18.5 Col2a1-Cre; slc26a $2^{\text {fl/fl }}$ growth plates compared with those of Col2a1-Cre; slc26a $2^{f l+}$ littermate controls (Fig. $8 \mathrm{H}, \mathrm{J}$ ). More importantly, we detected upregulated expression of ATF6 and enhanced phosphorylation of ERK1/2 by immunostaining on sections of E18.5 Col2a1-Cre; slc26a2 ${ }^{\text {fl/fl }}$ growth plates, which suggests a common mechanism underlying skeletal abnormalities shared by Col2a1-Cre; slc26a2 fl/fl and slc26a2 $2^{-1-}$ mice (Fig. 8I-J). Given the fact that Col2a1-Cre; slc26a2 $2^{f / f l}$ mice recapitulate the perinatal lethality and skeletal phenotypes of slc26a2 ${ }^{-/-}$mice, we conclusively demonstrate that SLC26A2 shares no redundancy with other chondrocyte-expressing sulfate transporters, and the lethality of global SLC26A2 deletion mostly results from skeletonspecific pathogenesis. 
A

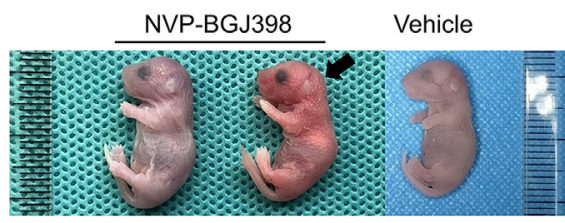

B
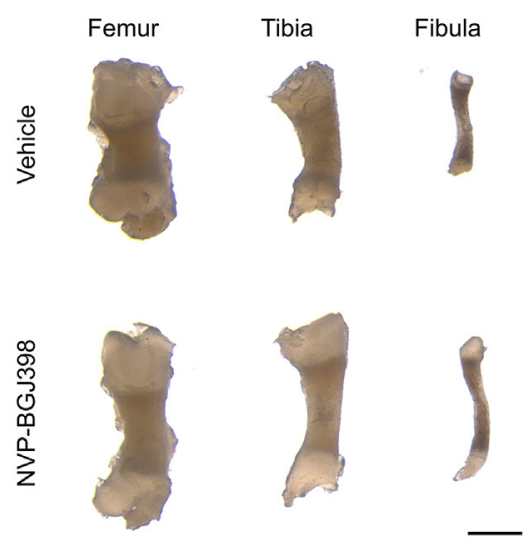

$\mathrm{F}$

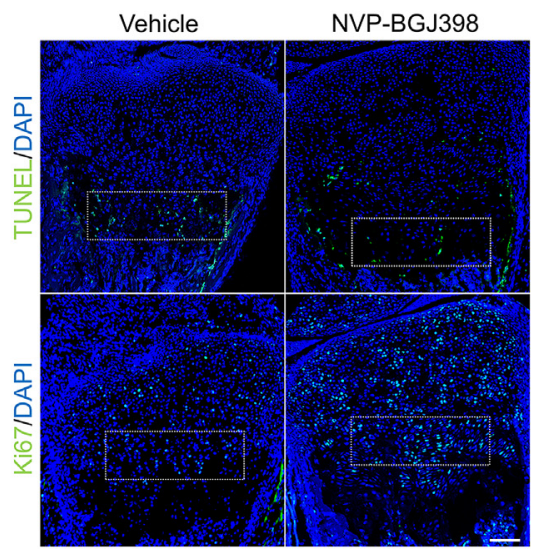

C

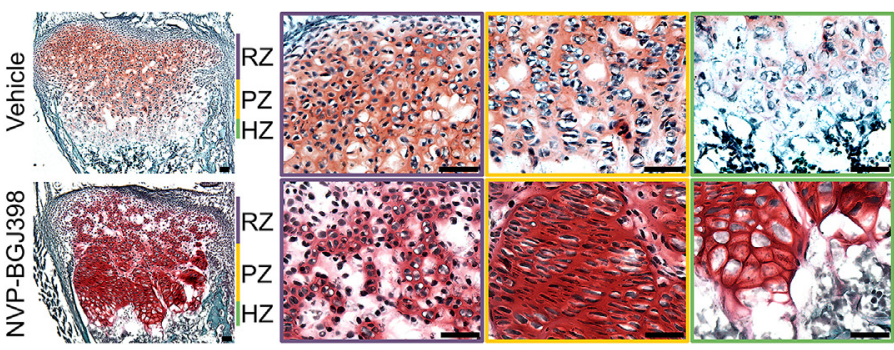

D

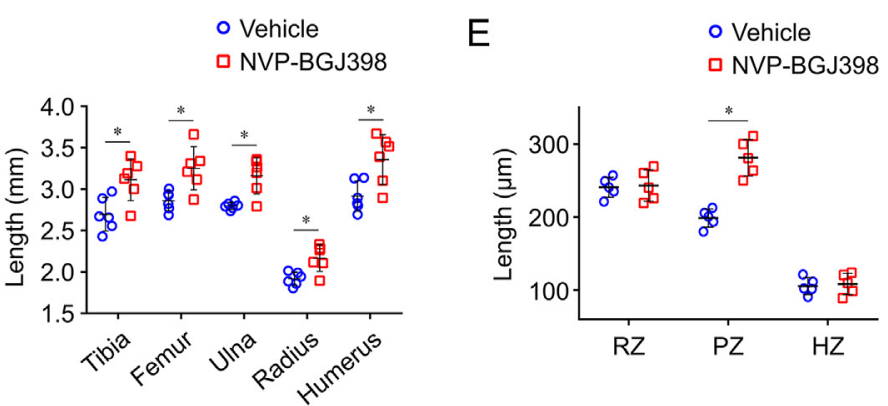

G

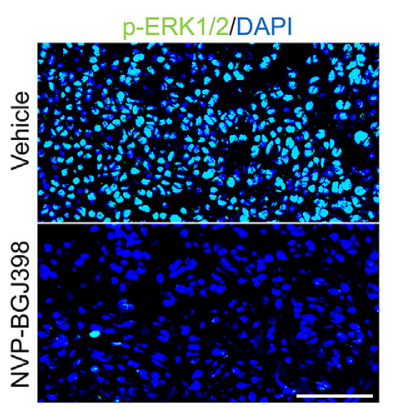

$\mathrm{H}$

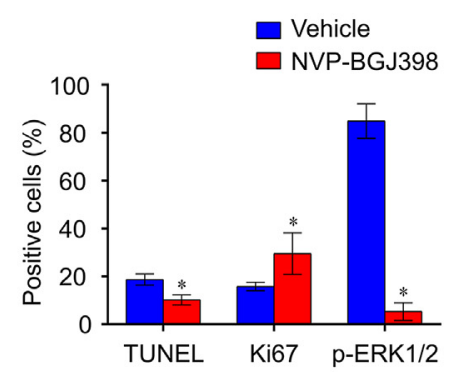

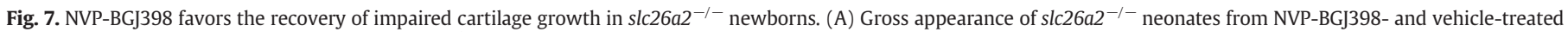

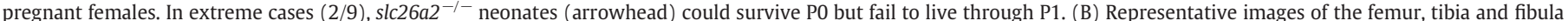

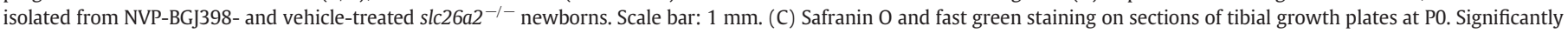

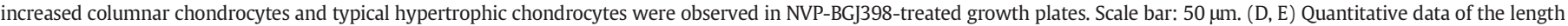

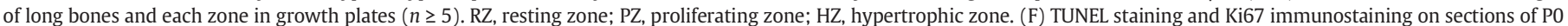

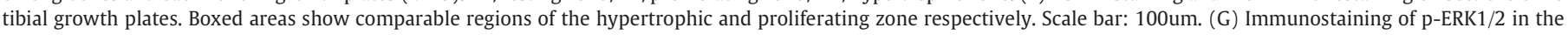

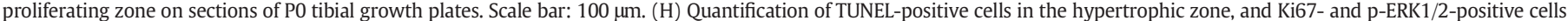
in the proliferating zone $(\mathrm{n}=3)$. For all the above-mentioned statistical analyses, significance was determined by Student's $t$-test, and results were shown as mean \pm SD. *: $\mathrm{p}<.05$.

\section{Discussion}

Research on ACG1B and AO2, two most severe forms of SLC26A2related chondrodysplasias, remains in its infancy due to lack of proper mouse models and thus to hamper therapeutic development. In this work, we carried out the first systemic investigation into the pathogenesis of SLC26A2-deficient lethal chondrodysplasias, the biological functions of SLC26A2 in chondrocytes and its skeleton-specific pathology by analyzing slc26a2 $2^{-1-}$ and Col2a1-Cre; slc26a2 $2^{f l / f l}$ mouse lines. Through our effects to elucidate the disassociated genotypephenotype correlations of SLC26A2-related conditions by identifying unknown pathogenic factors, a previously unrecognized role of SLC26A2 in collagen secretion and a potential therapeutic target are together addressed.

We first studied ACG1B and AO2 by generating and analyzing the slc26a2 $2^{-1-}$ mouse model. All the slc26a2 $2^{-/-}$newborns died perinatally, which is the most distinctive difference between ACG1B and AO2 and other SLC26A2-related chondrodysplasias. The severity of skeletal deformities observed in slc26a2 $2^{-1-}$ mice falls in a range between ACG1B and AO2. ACG1B leads to a more severe underossification of the skeleton in human, such as complete lack of ossification of the vertebral bodies and ischium, than AO2 [46,47]. slc26a2 ${ }^{-1-}$ mice shared common clinical features with human ACG1B and AO2, whereas the thickened soft tissue of the neck as observed in slc26a2 $2^{-1-}$ mice is usually seen in ACG1B and less in AO2 [46]. The slc26a2 ${ }^{-1-}$ mice do not exhibit cleft palate, a constant feature of human AO2 but not ACG1B [25]. At the cellular level, the morphology of slc26a2 $2^{-1-}$ growth plate is much more severely altered than that of $d t d$ mice. Uniquely, we observed multiple cystic acellular areas within the ECM of slc26a2 $2^{-1-}$ growth plates which have been found typical in cartilage sections of patients with ACG1B and AO2 $[17,25,26]$. Particularly, these areas were completely devoid of collagen disposition in slc26a2 $2^{-1-}$ mice. It has been demonstrated that an 
A

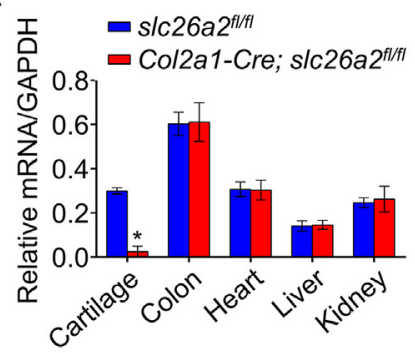

B

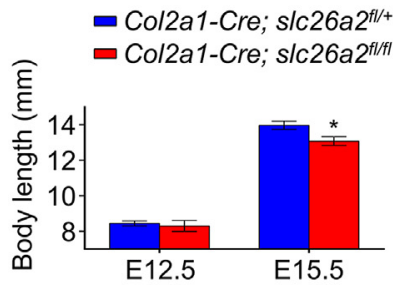

C
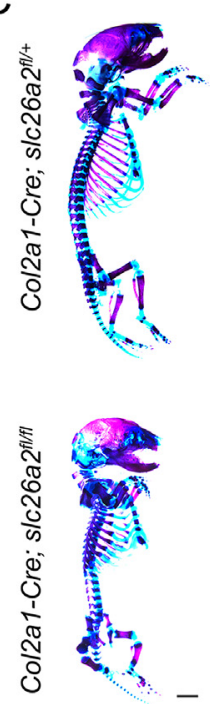

$\mathrm{D}$

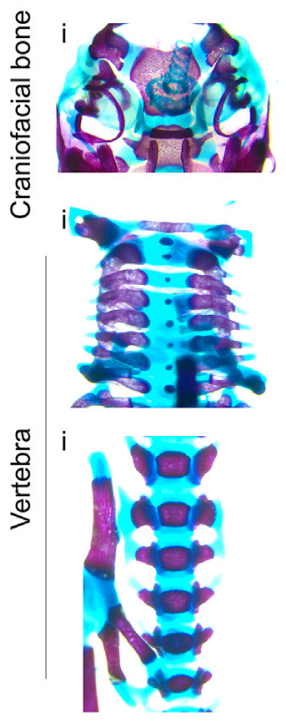

E

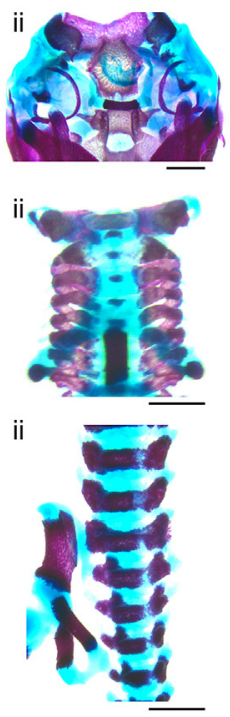

$\mathrm{F}$
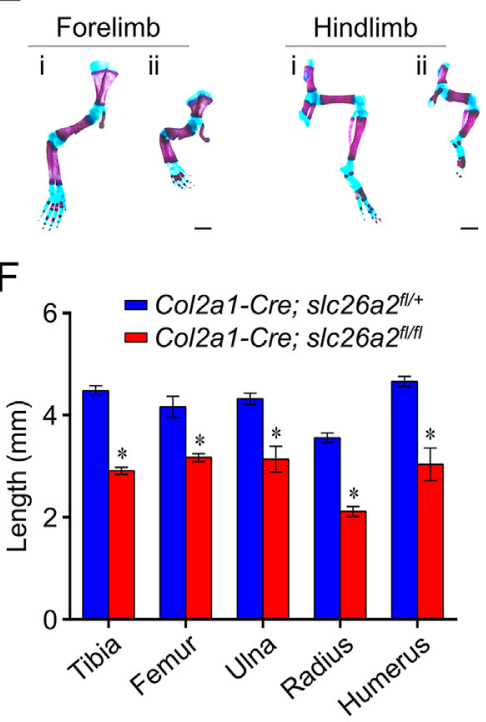

G

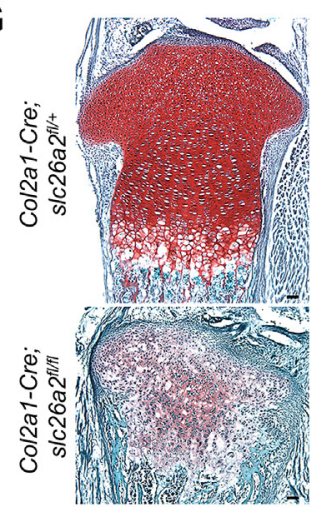

$\mathrm{H}$

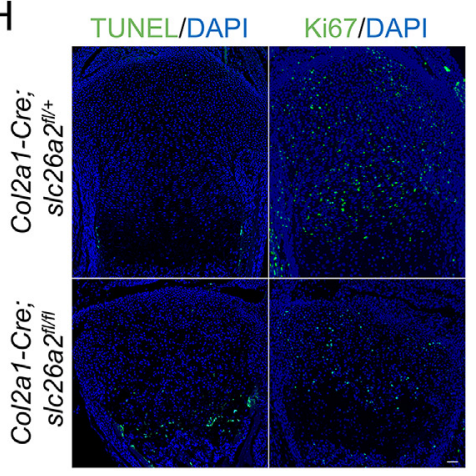

$J$

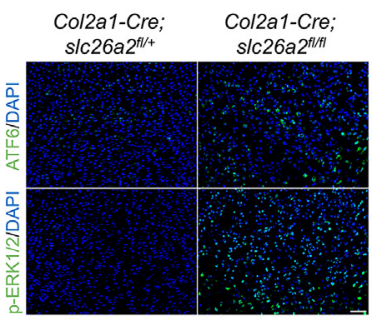

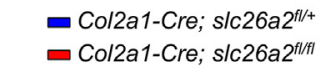

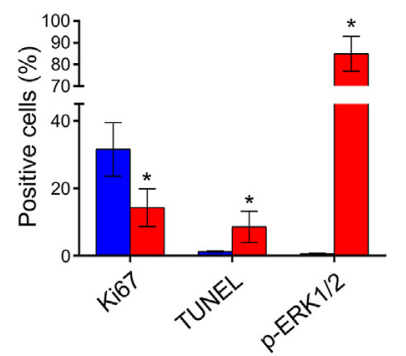

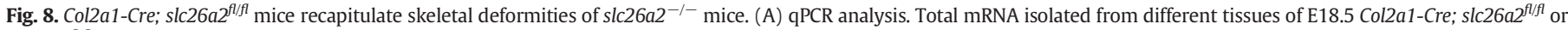

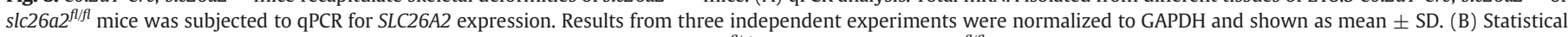

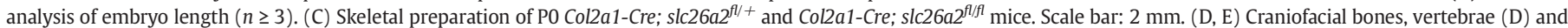

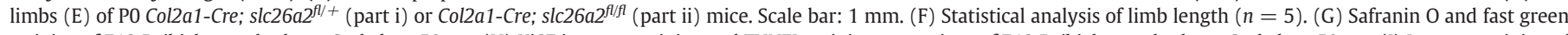

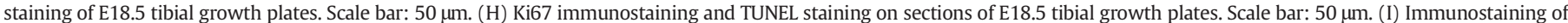

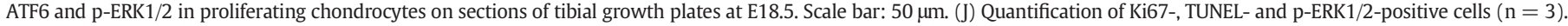
For all the above-mentioned statistical analyses, significance was determined by Student's $t$-test, and results were shown as mean \pm S.D. ${ }^{*}: \mathrm{p}<.05$.

abnormal extracellular structure, the ring around chondrocytes formed by lamellar materials, was found in the cartilage of patients with ACG1B, AO2 and DTD $[25,26]$. In some cases, this structure is called the "collagen ring" based on its morphology [47]. In concert with this, in slc26a2 ${ }^{-1-}$ mice, a large amount of collagen appears in the cytoplasm of chondrocytes whereas a relatively small amount of collagen is concentrated around chondrocytes among areas of normal cellularity. The recapitulation of human clinical features and defective morphogenesis of growth plates in slc26a2 $2^{-1-}$ mice justifies the potential applicability of our findings to human conditions.

Studies on mouse models with secretory defects have depicted a complex pathogenic paradigm of inherited skeletal diseases, involving selective activation and vastly different actions of discrete UPR signaling modules in each corresponding condition [30,64-66]. It is interesting to find that the ATF6 arm of the UPR is preferentially elicited by defective secretion of ColII/IX in slc26a2 ${ }^{-1-}$ chondrocytes. Likewise, in mouse models of metaphyseal chondrodysplasia type Schmid (MCDS), intracellularly retained mutant ColX triggers the PERK arm of the UPR, whereas misfolding of mutant Coll with a G610C substitution invokes an unusual stress response by which the conventional UPR sensor BiP or Xbp1 are not induced in a mouse model of osteogenesis imperfecta $[30,64,67]$. Together, our study and others indicate that the employment of UPR arms is largely stressor-dependent. It has been reported that ATF6 is constantly expressed in growth plate chondrocytes and favors chondrocyte hypertrophy in vitro by acting as the co-factor of Runx2 and modulating Ihh and PTHrP signaling during cartilage development $[35,68]$. However, ATF6 knockout mice display no apparent skeletal phenotype whereas ablation of either ATF4 or XBP1 in mice causes evident skeletal defects, suggesting that ATF6 among other constantly expressed UPR components is not obligatory for chondrocyte growth under physiological conditions $[31,36,53]$. The only existing in vivo functional analysis of ATF6 in stressed chondrocytes is a recent report where deleting ATF6 in MCDS mice expressing mutant ColX with an N617K substitution worsens the phenotype through an unknown molecular mechanism [53]. Notably, we found that upregulated FGFR3 together with enhanced signaling was caused by and dominated the actions of the ATF6 arm in slc26a2 ${ }^{-/-}$chondrocytes. FGFR3 signaling plays an essential role in cartilage development by negatively regulating chondrocyte proliferation [69]. Mice expressing mutant activated FGFR3 and slc26a2 $2^{-1-}$ mice share common phenotypic features of shortened long bones and premature closure of the neurocentral synchondroses of vertebrae [70-73]. In addition to enhanced phosphorylation of ERK1/2 and STAT1 as putative mediators of FGFR3 signaling [49], Sox9 was continually present, albeit at low levels, in slc26a2 $2^{-1-}$ hypertrophic 
chondrocytes, whose expression was already shut down in those of controls. Similarly, a recent study shows that hyperactive FGFR3 prevents the downregulation of Sox9 in differentiating chondrocytes and thus to hinder chondrocyte maturation [74]. These phenotypic and molecular similarities shared by slc26a2 $2^{-1-}$ and FGFR3 mutant mice are consistent with the domination of FGFR3 signaling in the pathology of SLC26A2 deficiency. Based on our study and others, here we propose a potential pathological paradigm of ATF6-FGFR3 in slc26a2 ${ }^{-1-}$ chondrocytes: to handle transient ECM protein load, UPR sensors may employ inhibitory pathways to ensure proliferative inactivation, thereby protecting chondrocytes from further increased matrix protein load and benefiting the recovery of proteostasis [53,54,75], whereas overwhelmingly activated ATF6 arm of the UPR by SLC26A2 ablation paradoxically leads to impaired cartilage growth through the exceeding enhancement of FGFR3 signaling. However, whether FGFR3 is a universal downstream mediator of ATF6 needs to be further confirmed in other ATF6-related disease models.

Fetuses with ACG1B and AO2 show readily severe skeletal abnormalities at an early gestational age of 12 weeks when the perinatal diagnosis could be made by sonographic examination $[46,76]$. It is rather difficult to carry out medical management and surgical repair on human chondrodysplasias during pregnancy. Thus far, there has been no alternative therapy under investigation but to provide palliative care for viable ACG1B and AO2 newborns [46,47]. Drug intervention during pregnancy has been proved practical and effective to ameliorate the retarded skeletal development and rescue postnatal death in mouse models mimicking human chondrodysplasias [77-79]. To our best knowledge, we conducted the first preclinical study with NVP-BGJ398 in SLC26A2-related conditions. NVP-BGJ398 emerges as a promising treatment for FGFR3-related tumors and chondrodysplasias [55] and is currently in several phase 2 clinical trials (ClinicalTrials. govNCT02160041, NCT02159066 and NCT01975701). Targeting FGFR3 signaling with NVP-BGJ398 or the inhibitor of downstream effector could favorably modulate the growth of slc26a2 ${ }^{-1-}$ cartilage explant cultures. Furthermore, we tested NVP-BGJ398 with timed pregnant females and observed significantly increased number of typical columnar chondrocytes and improved linear bone growth in slc26a2 ${ }^{-1-}$ newborns. In line with the morphological improvement, the altered balance of chondrocyte proliferation and apoptosis was evidently restored. Consistent with the previous study [55], NVP-BGJ398 could also efficiently suppress FGFR3 signaling in slc26a2 $2^{-1-}$ chondrocytes, indicated by significantly reduced phosphorylation of ERK1/2. Although NVP-BGJ398 did not fully rectify skeletal abnormalities of slc26a $2^{-1-}$ newborns, its therapeutic effects on slc26a2 $2^{-/-}$pathological features justify suppression of hyperactive FGFR3 signaling as a rationale pharmacological strategy for SLC26A2-related chondrodysplasias. Therefore, FGFR3 inhibitors could be repurposed to treat ACG1B and AO2, and our slc26a2 ${ }^{-1-}$ mouse model is well-suited to test other FGFR3-targeted therapeutic approaches for ACG1B and AO2.

In the present study, we also demonstrate for the first time that skeleton-specific inactivation of SLC26A2 is sufficient to cause perinatal lethality, independent of contributions from other non-skeletal tissues expressing SLC26A2. Notwithstanding the shared neonatal death and phenotypic features, Col2a1-Cre; slc26a2 $2^{f l f l}$ mice show somewhat less defective bone elongation compared with slc26a2 $2^{-1-}$ mice. A possible explanation is that Col2a1-Cre; slc26a $2^{f l / f l}$ mice do not lose SLC26A2 expression in all skeletal cells; Cre-mediated recombination only takes place in a portion of osteoblastic cells that also contribute to bone growth [80]. Indeed, it has been reported that SLC26A2 shared a similar expression pattern with Col1a1 in the differentiating osteoblastic precursor cells [81], and osteoblasts expressing mutant SLC26A2 exhibited impaired sulfate uptake [4]. Thus, SLC26A2 may play a potential role in osteoblasts, which remains to be further explored.

Collectively, the present study has expanded our view of the sulfate transporter SLC26A2 to the unexpected field of collagen secretion and proteostasis. Our findings will facilitate the understanding of genotype-phenotype relationships of SLC26A2-related chondrodysplasias and accelerate the development of drug therapy against ACG1B and $\mathrm{AO} 2$.

\section{Conflict of interest}

There is no conflict of interests to report, and all authors have conformed to all the editorial policies of EBioMedicine.

\section{Funding sources}

This work was supported by National Natural Science Foundation of China (81871743, 81730065 and 81772377$)$. The funder had no role in study design, data collection, data analysis, interpretation, or writing of the report.

\section{Declaration of interests}

The authors declare no conflict of interest.

\section{Author contributions}

Chao Zheng, Qiang Jie, Zhuojing Luo and Liu Yang designed research; Chao Zheng, Xisheng Lin, Xiaolong Xu and Cheng Wang performed experiments; Cheng Wang and Jinru Zhou contributed new reagents and analytic tools; Jinru Zhou, Bo Gao, Jing Fan, Weiguang Lu and Yaqian Hu analyzed data; and Chao Zheng and Liu Yang wrote the paper. All authors reviewed and approved the manuscript.

\section{Acknowledgements}

We thank Dr. Kathryn S.E.Cheah for the Col10a1 antibody and Dr. Linyu Lu for Vasa-Cre mice.

\section{Appendix A. Supplementary data}

Supplementary data to this article can be found online at https://doi. org/10.1016/j.ebiom.2019.01.010.

\section{References}

[1] Soares da Costa D, Reis RL, Pashkuleva I. Sulfation of Glycosaminoglycans and its Implications in Human Health and Disorders. Annu Rev Biomed Eng 2017:19:1-26.

[2] Mueller JW, Gilligan LC, Idkowiak J, Arlt W, Foster PA. The regulation of steroid action by sulfation and desulfation. Endocr Rev 2015;36(5):526-63.

[3] Yang YS, Wang CC, Chen BH, Hou YH, Hung KS, Mao YC. Tyrosine sulfation as a protein post-translational modification. Molecules 2015;20(2):2138-64.

[4] Forlino A, Piazza R, Tiveron C, Della Torre S, Tatangelo L, Bonafe L, et al. A diastrophic dysplasia sulfate transporter (SLC26A2) mutant mouse: morphological and biochemical characterization of the resulting chondrodysplasia phenotype. Hum Mol Genet 2005;14(6):859-71.

[5] Cortes M, Baria AT, Schwartz NB. Sulfation of chondroitin sulfate proteoglycans is necessary for proper Indian hedgehog signaling in the developing growth plate. Development 2009;136(10):1697-706.

[6] Kluppel M, Wight TN, Chan C, Hinek A, Wrana JL. Maintenance of chondroitin sulfation balance by chondroitin-4-sulfotransferase 1 is required for chondrocyte development and growth factor signaling during cartilage morphogenesis. Development 2005;132(17):3989-4003.

[7] Bullock SL, Fletcher JM, Beddington RS, Wilson VA. Renal agenesis in mice homozygous for a gene trap mutation in the gene encoding heparan sulfate 2sulfotransferase. Genes Dev 1998;12(12):1894-906.

[8] Ratzka A, Kalus I, Moser M, Dierks T, Mundlos S, Vortkamp A. Redundant function of the heparan sulfate 6-O-endosulfatases Sulf1 and Sulf2 during skeletal development. Dev Dyn 2008;237(2):339-53.

[9] Ringvall M, Ledin J, Holmborn K, van Kuppevelt T, Ellin F, Eriksson I, et al. Defective heparan sulfate biosynthesis and neonatal lethality in mice lacking N-deacetylase/Nsulfotransferase-1. J Biol Chem 2000;275(34):25926-30.

[10] Frederick JP, Tafari AT, Wu SM, Megosh LC, Chiou ST, Irving RP, et al. A role for a lithium-inhibited Golgi nucleotidase in skeletal development and sulfation. Proc Natl Acad Sci U S A 2008;105(33):11605-12.

[11] Haila S, Hastbacka J, Bohling T, Karjalainen-Lindsberg ML, Kere J. Saarialho-Kere U. SLC26A2 (diastrophic dysplasia sulfate transporter) is expressed in developing and mature cartilage but also in other tissues and cell types. J Histochem Cytochem 2001;49(8):973-82. 
[12] Park M, Ohana E, Choi SY, Lee MS, Park JH, Muallem S. Multiple roles of the SO4(2-)/ $\mathrm{Cl}-/ \mathrm{OH}$ - exchanger protein Slc26a2 in chondrocyte functions. J Biol Chem 2014;289 (4):1993-2001.

[13] Stenson PD, Mort M, Ball EV, Evans K, Hayden M, Heywood S, et al. The human gene mutation database: towards a comprehensive repository of inherited mutation data for medical research, genetic diagnosis and next-generation sequencing studies. Hum Genet 2017;136(6):665-77.

[14] Rossi A, Superti-Furga A. Mutations in the diastrophic dysplasia sulfate transporter (DTDST) gene (SLC26A2): 22 novel mutations, mutation review, associated skeletal phenotypes, and diagnostic relevance. Hum Mutat 2001;17(3):159-71.

[15] Cai T, Yang L, Cai W, Guo S, Yu P, Li J, et al. Dysplastic spondylolysis is caused by mutations in the diastrophic dysplasia sulfate transporter gene. Proc Natl Acad Sci U S A 2015;112(26):8064-9.

[16] Gualeni B, Facchini M, De Leonardis F, Tenni R, Cetta G, Viola M, et al. Defective proteoglycan sulfation of the growth plate zones causes reduced chondrocyte proliferation via an altered Indian hedgehog signalling. Matrix Biol 2010;29(6):453-60.

[17] Cornaglia AI, Casasco A, Casasco M, Riva F, Necchi V. Dysplastic histogenesis of cartilage growth plate by alteration of sulphation pathway: a transgenic model. Connect Tissue Res 2009;50(4):232-42.

[18] Mertz EL, Facchini M, Pham AT, Gualeni B, De Leonardis F, Rossi A, et al. Matrix disruptions, growth, and degradation of cartilage with impaired sulfation. J Biol Chem 2012;287(26):22030-42.

[19] Gualeni B, de Vernejoul MC, Marty-Morieux C, De Leonardis F, Franchi M, Monti L, et al. Alteration of proteoglycan sulfation affects bone growth and remodeling. Bone 2013;54(1):83-91.

[20] Karniski LP. Mutations in the diastrophic dysplasia sulfate transporter (DTDST) gene: correlation between sulfate transport activity and chondrodysplasia phenotype. Hum Mol Genet 2001;10(14):1485-90.

[21] Dwyer E, Hyland J, Modaff P, Pauli RM. Genotype-phenotype correlation in DTDST dysplasias: Atelosteogenesis type II and diastrophic dysplasia variant in one family. Am J Med Genet A 2010;152A(12):3043-50.

[22] Rossi A, van der Harten HJ, Beemer FA, Kleijer WJ, Gitzelmann R, Steinmann B, et al. Phenotypic and genotypic overlap between atelosteogenesis type 2 and diastrophic dysplasia. Hum Genet 1996;98(6):657-61.

[23] Rossi A, Bonaventure J, Delezoide AL, Cetta G, Superti-Furga A. Undersulfation of proteoglycans synthesized by chondrocytes from a patient with achondrogenesis type 1B homozygous for an L483P substitution in the diastrophic dysplasia sulfate transporter. J Biol Chem 1996;271(31):18456-64.

[24] Hastbacka J, Superti-Furga A, Wilcox WR, Rimoin DL, Cohn DH, Lander ES. Atelosteogenesis type II is caused by mutations in the diastrophic dysplasia sulfate-transporter gene (DTDST): evidence for a phenotypic series involving three chondrodysplasias. Am J Hum Genet 1996;58(2):255-62.

[25] Newbury-Ecob R. Atelosteogenesis type 2. J Med Genet 1998;35(1):49-53.

[26] Superti-Furga A. Achondrogenesis type 1B. J Med Genet 1996;33(11):957-61.

[27] Myllyharju J, Kivirikko KI. Collagens, modifying enzymes and their mutations in humans, flies and worms. Trends Genet 2004;20(1):33-43.

[28] Annunen S, Paassilta P, Lohiniva J, Perala M, Pihlajamaa T, Karppinen J, et al. An allele of COL9A2 associated with intervertebral disc disease. Science 1999;285(5426): 409-12.

[29] Gawron K. Endoplasmic reticulum stress in chondrodysplasias caused by mutations in collagen types II and X. Cell Stress Chaperones 2016;21(6):943-58.

[30] Wang C, Tan Z, Niu B, Tsang KY, Tai A, Chan WCW, et al. Inhibiting the integrated stress response pathway prevents aberrant chondrocyte differentiation thereby alleviating chondrodysplasia. elife 2018;7.

[31] Cameron TL, Gresshoff IL, Bell KM, Pirog KA, Sampurno L, Hartley CL, et al. Cartilagespecific ablation of XBP1 signaling in mouse results in a chondrodysplasia characterized by reduced chondrocyte proliferation and delayed cartilage maturation and mineralization. Osteoarthr Cartil 2015;23(4):661-70.

[32] Walter P, Ron D. The unfolded protein response: from stress pathway to homeostatic regulation. Science 2011;334(6059):1081-6.

[33] Marciniak SJ, Ron D. Endoplasmic reticulum stress signaling in disease. Physiol Rev 2006;86(4):1133-49.

[34] Tabas I, Ron D. Integrating the mechanisms of apoptosis induced by endoplasmic reticulum stress. Nat Cell Biol 2011;13(3):184-90.

[35] Guo F, Han X, Wu Z, Cheng Z, Hu Q, Zhao Y, et al. ATF6a, a Runx2-activable transcription factor, is a new regulator of chondrocyte hypertrophy. J Cell Sci 2016;129(4): 717-28.

[36] Wang W, Lian N, Li L, Moss HE, Wang W, Perrien DS, et al. Atf4 regulates chondrocyte proliferation and differentiation during endochondral ossification by activating Ihh transcription. Development 2009;136(24):4143-53.

[37] Horiuchi K, Tohmonda T, Morioka H. The unfolded protein response in skeletal development and homeostasis. Cell Mol Life Sci 2016;73(15):2851-69.

[38] Gallardo T, Shirley L, John GB, Castrillon DH. Generation of a germ cell-specific mouse transgenic Cre line, Vasa-Cre. Genesis 2007;45(6):413-7.

[39] Yan B, Zhang Z, Jin D, Cai C, Jia C, Liu W, et al. mTORC1 regulates PTHrP to coordinate chondrocyte growth, proliferation and differentiation. Nat Commun 2016;7:11151.

[40] Yang L, Tsang KY, Tang HC, Chan D, Cheah KS. Hypertrophic chondrocytes can become osteoblasts and osteocytes in endochondral bone formation. Proc Natl Acad Sci U S A 2014;111(33):12097-102.

[41] Day TF, Guo X, Garrett-Beal L, Yang Y. Wnt/beta-catenin signaling in mesenchymal progenitors controls osteoblast and chondrocyte differentiation during vertebrate skeletogenesis. Dev Cell 2005;8(5):739-50.

[42] Gao B, Hu J, Stricker S, Cheung M, Ma G, Law KF, et al. A mutation in Ihh that causes digit abnormalities alters its signalling capacity and range. Nature 2009;458(7242): 1196-200.
[43] Wuelling M, Vortkamp A. Cartilage explant cultures. Methods Mol Biol 2014;1130: 89-97.

[44] Kronenberg HM. Developmental regulation of the growth plate. Nature 2003;423 (6937):332-6.

[45] Yang Y. Skeletal morphogenesis during embryonic development. Crit Rev Eukaryot Gene Expr 2009;19(3):197-218

[46] Bonafe L, Mittaz-Crettol L, Ballhausen D, Superti-Furga A. Achondrogenesis Type 1B. In: Adam MP, Ardinger HH, Pagon RA, Wallace SE, Bean LJH, Stephens K, et al, editors. GeneReviews((R)); 1993 Seattle (WA).

[47] Bonafe L, Mittaz-Crettol L, Ballhausen D, Superti-Furga A. Atelosteogenesis Type 2. In: Adam MP, Ardinger HH, Pagon RA, Wallace SE, Bean LJH, Stephens K, et al, editors. GeneReviews((R)); 1993 Seattle (WA).

[48] Rossi A, Kaitila I, Wilcox WR, Rimoin DL, Steinmann B, Cetta G, et al. Proteoglycan sulfation in cartilage and cell cultures from patients with sulfate transporter chondrodysplasias: relationship to clinical severity and indications on the role of intracellular sulfate production. Matrix Biol 1998;17(5):361-9.

[49] Kozhemyakina E, Lassar AB, Zelzer E. A pathway to bone: signaling molecules and transcription factors involved in chondrocyte development and maturation. Development 2015;142(5):817-31.

[50] Newman B, Wallis GA. Skeletal dysplasias caused by a disruption of skeletal patterning and endochondral ossification. Clin Genet 2003;63(4):241-51.

[51] Kilgore JA, Dolman NJ, Davidson MW. A review of reagents for fluorescence microscopy of cellular compartments and structures, Part II: reagents for non-vesicular organelles. Curr Protoc Cytom 2013;66:31 Unit 12.

[52] Yasoda A, Komatsu Y, Chusho H, Miyazawa T, Ozasa A, Miura M, et al. Overexpression of CNP in chondrocytes rescues achondroplasia through a MAPK-dependent pathway. Nat Med 2004;10(1):80-6

[53] Forouhan M, Mori K, Boot-Handford RP. Paradoxical roles of ATF6alpha and ATF6beta in modulating disease severity caused by mutations in collagen X. Matrix Biol 2018;70:50-71.

[54] Hetz C. The unfolded protein response: controlling cell fate decisions under ER stress and beyond. Nat Rev Mol Cell Biol 2012;13(2):89-102.

[55] Komla-Ebri D, Dambroise E, Kramer I, Benoist-Lasselin C, Kaci N, Le Gall C, et al. Tyrosine kinase inhibitor NVP-BGJ398 functionally improves FGFR3-related dwarfism in mouse model. J Clin Invest 2016;126(5):1871-84.

[56] Murakami S, Balmes G, McKinney S, Zhang Z, Givol D, de Crombrugghe B. Constitutive activation of MEK1 in chondrocytes causes Stat1-independent achondroplasialike dwarfism and rescues the Fgfr3-deficient mouse phenotype. Genes Dev 2004; 18(3):290-305

[57] Spyroglou A, Bozoglu T, Rawal R, De Leonardis F, Sterner C, Boulkroun S, et al. Diastrophic dysplasia sulfate transporter (SLC26A2) is expressed in the adrenal cortex and regulates aldosterone secretion. Hypertension 2014;63(5):1102-9.

[58] Dimberg LY, Towers CG, Behbakht K, Hotz T], Kim J, Fosmire S, et al. A Genome-Wide Loss-of-Function Screen Identifies SLC26A2 as a Novel Mediator of TRAIL Resistance. Mol Cancer Res 2017;15(4):382-94.

[59] Inoue A, Okamoto K, Fujino Y, Nakagawa T, Muguruma N, Sannomiya K, et al. B-RAF mutation and accumulated gene methylation in aberrant crypt foci (ACF), sessile serrated adenoma/polyp (SSA/P) and cancer in SSA/P. Br J Cancer 2015;112(2): 403-12.

[60] Yusa A, Miyazaki K, Kimura N, Izawa M, Kannagi R. Epigenetic silencing of the sulfate transporter gene DTDST induces sialyl Lewisx expression and accelerates proliferation of colon cancer cells. Cancer Res 2010;70(10):4064-73.

[61] Comelli EM, Lariani S, Zwahlen MC, Fotopoulos G, Holzwarth JA, Cherbut C, et al. Biomarkers of human gastrointestinal tract regions. Mamm Genome 2009;20(8): 516-27.

[62] Simmons DG, Rakoczy J, Jefferis J, Lourie R, McIntyre HD, Dawson PA. Human placental sulfate transporter mRNA profiling from term pregnancies identifies abundant SLC13A4 in syncytiotrophoblasts and SLC26A2 in cytotrophoblasts. Placenta 2013; 34(4):381-4

[63] Dawson PA, Rakoczy J, Simmons DG. Placental, renal, and ileal sulfate transporter gene expression in mouse gestation. Biol Reprod 2012;87(2):43.

[64] Mirigian LS, Makareeva E, Mertz EL, Omari S, Roberts-Pilgrim AM, Oestreich AK, et al. Osteoblast malfunction caused by cell stress response to Procollagen misfolding in alpha2(I)-G610C mouse model of osteogenesis imperfecta. J Bone Miner Res 2016; 31(8):1608-16.

[65] Hotamisligil GS, Davis RJ. Cell signaling and stress responses. Cold Spring Harb Perspect Biol 2016;8(10).

[66] Cameron TL, Bell KM, Gresshoff IL, Sampurno L, Mullan L, Ermann J, et al. XBP1-independent UPR pathways suppress C/EBP-beta mediated chondrocyte differentiation in ER-Stress related skeletal disease. PLoS Genet 2015;11(9): e1005505.

[67] Mullan LA, Mularczyk EJ, Kung LH, Forouhan M, Wragg JM, Goodacre R, et al. Increased intracellular proteolysis reduces disease severity in an ER stress-associated dwarfism. J Clin Invest 2017;127(10):3861-5.

[68] Xiong Z, Jiang R, Zhang P, Han X, Guo FJ. Transmission of ER stress response by ATF6 promotes endochondral bone growth. J Orthop Surg Res 2015;10:141.

[69] Foldynova-Trantirkova S, Wilcox WR, Krejci P. Sixteen years and counting: the current understanding of fibroblast growth factor receptor 3 (FGFR3) signaling in skeletal dysplasias. Hum Mutat 2012;33(1):29-41.

[70] Zhou S, Xie Y, Tang J, Huang J, Huang Q, Xu W, et al. FGFR3 deficiency causes multiple chondroma-like lesions by Upregulating Hedgehog Signaling. PLoS Genet 2015;11 (6):e1005214.

[71] Wang Y, Spatz MK, Kannan K, Hayk H, Avivi A, Gorivodsky M, et al. A mouse model for achondroplasia produced by targeting fibroblast growth factor receptor 3 . Proc Natl Acad Sci U S A 1999;96(8):4455-60. 
[72] Chen L, Li C, Qiao W, Xu X, Deng C. A Ser(365)->Cys mutation of fibroblast growth factor receptor 3 in mouse downregulates Ihh/PTHrP signals and causes severe achondroplasia. Hum Mol Genet 2001;10(5):457-65.

[73] Matsushita T, Wilcox WR, Chan YY, Kawanami A, Bukulmez H, Balmes G, et al. FGFR3 promotes synchondrosis closure and fusion of ossification centers through the MAPK pathway. Hum Mol Genet 2009;18(2):227-40.

[74] Zhou ZQ, Ota S, Deng C, Akiyama H, Hurlin PJ. Mutant activated FGFR3 impairs endochondral bone growth by preventing SOX9 downregulation in differentiating chondrocytes. Hum Mol Genet 2015;24(6):1764-73.

[75] Wilson DG, Phamluong K, Li L, Sun M, Cao TC, Liu PS, et al. Global defects in collagen secretion in a Mia3/TANGO1 knockout mouse. J Cell Biol 2011;193(5):935-51.

[76] Fernandez-Aguilar S, Noel JC, Van Regemorter N, Superti-Furga A, Bonafe L, Donner C. Diagnosis of atelosteogenesis type II after a routine echography at 12 weeks' pregnancy. Prenat Diagn 2005;25(8):717-8.

[77] Xie Y, Su N, Jin M, Oi H, Yang J, Li C, et al. Intermittent PTH (1-34) injection rescues the retarded skeletal development and postnatal lethality of mice mimicking human achondroplasia and thanatophoric dysplasia. Hum Mol Genet 2012;21(18): 3941-55.

[78] Monti L, Paganini C, Lecci S, De Leonardis F, Hay E, Cohen-Solal M, et al. Nacetylcysteine treatment ameliorates the skeletal phenotype of a mouse model of diastrophic dysplasia. Hum Mol Genet 2015;24(19):5570-80.

[79] Ornitz DM, Legeai-Mallet L. Achondroplasia: development, pathogenesis, and therapy. Dev Dyn 2017;246(4):291-309.

[80] Ono N, Ono W, Nagasawa T, Kronenberg HM. A subset of chondrogenic cells provides early mesenchymal progenitors in growing bones. Nat Cell Biol 2014;16 (12):1157-67

[81] Kobayashi T, Sugimoto T, Saijoh K, Fukase M, Chihara K. Cloning of mouse diastrophic dysplasia sulfate transporter gene induced during osteoblast differentiation by bone morphogenetic protein-2. Gene 1997;198(1-2):341-9. 